\title{
Comparative Analysis of the Measured and Extrapolated Wind Shear Distributions at Murtala Mohammed and Port Harcourt International Airports, Nigeria
}

\author{
Onwuadiochi, I. C..$^{*} \quad$ Ijioma, M. A. ${ }^{1} \quad$ Mage, J. O. $^{2} \quad$ Ozoemene, M. L. $^{1}$ \\ 1.Department of Geography and Meteorology, Nnamdi Azikiwe University, Awka, Anambra State, Nigeria \\ 2.Department of Geography, Benue State University, Makurdi, Nigeria
}

\begin{abstract}
The persistent gory incidents, accidents, loss of huge amount of money and outcry by the aviation industry for accurate weather observation and forecast have been of utmost worry to so many researchers. In a bid to curbing these aviation weather challenges, researchers have discovered that wind shear impacts greatly on flight operations in the airports, and are working towards ameliorating the effects. This study analyzed the distribution of measured and extrapolated wind shear at Murtala Mohammed and Port Harcourt International Airports, Nigeria. The annual time series secondary data used for this study were sourced from Nigerian Airspace Management Agency (NAMA) at Murtala Mohammed and Port Harcourt International Airports and Nigerian Meteorological Agency (NIMET). Power Law Model was used to extrapolate wind shear at 20m above ground level to $50 \mathrm{~m}$ and $100 \mathrm{~m}$ above ground level for the both airports. The data were compiled and presented in tables and charts for easy understanding and further analysis. The statistical techniques employed in achieving the research target were descriptive statistics (such as mean, standard deviations, coefficient of variation and charts). The study shows that wind shear increases with altitude at the study areas due to decrease in frictional resistance which is more pronounced close to the ground. A similarity in the distribution pattern of wind shear at $20 \mathrm{~m}, 50 \mathrm{~m}$ and $100 \mathrm{~m}$ above ground level at Murtala Mohammed International Airport was revealed by the study. The same similarity in the distribution pattern was also revealed at Port Harcourt International Airport. Evidently, the study reveals some temporal variations in the mean and standard deviation of wind shear in the study areas. The study also reveals based on the effects of wind shear and its resultant flight delays, diversions and cancellations that Port Harcourt International Airport with overall higher coefficient of variation (CV) is considered safer for flight landing. The study therefore, based on its findings, recommends subsequent training and retraining of Meteorologists, Pilots and Air Traffic Controllers on wind shear and related weather issues. The work also recommends that more studies should be carried out by the students and researchers on wind shear.
\end{abstract}

Keywords: Wind Shear, Comparative Analysis, Measured, Extrapolated, Distributions

DOI: $10.7176 / \mathrm{JEES} / 11-5-06$

Publication date:May $31^{\text {st }} 2021$

\section{Introduction}

The growth of aviation industry in Nigeria and the increased adoption of air transportation as one of the best means of transport have been obstructed by various weather hazards. There is a greater need for aviation weather forecasters to deliver quality forecasts (Weli \& Ifediba, 2014). Despite the relatively conducive weather of Nigeria compared to other countries (such as Mauritania, Somalia, Japan etc.), there has been a marked increase in the cases of recorded flight delay, diversion and cancellation, which in most cases, are attributed to poor weather conditions such as wind shear, thunderstorm, poor visibility, fog, dust haze and line squall (National Oceanic and Atmospheric Administration (NOAA), 2004; Sasse \& Hauf, 2003; Jones, 2004; Knetch, 2008; Weli \& Ifediba, 2014; Musa, 2014; Enete, Ajator, \& Nwoko, 2015; Onwuadiochi, Ijioma, \& Ozoemene, 2020).

The effects of wind shear on aircraft operations are countless and because of this, many researchers have defined it in various ways. For instance, Azad (2011) defined wind shear as a difference in wind speed and wind direction over a relatively short distance in the earth's atmosphere. Wind is, of course, a possible threat if not adequately considered in some specific fields. One of such fields is the designing of airport runways (Roberto, 2014). He stated that wind (crosswinds) blowing perpendicular to the runway may cause serious accidents, especially during landing and for small airplanes.

While there could be a vertical and horizontal wind shear, the former is critical factor in the determination of thunderstorm type and potential storm severity and airplane pilots regard the later as change in airspeed of 30 knots $(15 \mathrm{~m} / \mathrm{s})$ for light aircrafts, and near $45 \mathrm{knots}(22 \mathrm{~m} / \mathrm{s})$ for airliners at flight altitudes (Azad, 2011). Thus, low level wind shear can affect aircraft airspeed during takeoff and landing in disastrous ways, a condition which makes airliner pilots to be trained to avoid all microburst wind shear, that is, headwind loss in excess of 30 knots. In general, the wind shear events that present the greatest risk to aircraft are those associated with convective activity, specifically gust fronts and microburst, and such events have resulted in several major accidents 
involving large transport aircraft internationally (National Research Council, 1983; Azad, 2011).

Wind shear is caused by quite a number of factors, but ground surface roughness and obstacles stand out in bold relief. For example, land and sea breezes trigger wind shear. It is therefore not surprising that pilots passing across the coastlines are always very careful, because this is where the breezes predominate (Onwuadiochi, Ijioma, Ezenwaji, \& Obikwelu, 2020). During the daytime when the land gets heated by the radiation faster than the sea, and cooler air above the sea tends to move towards the land due to pressure variation; the process is known as the sea breeze. The same process is reversed during the nighttime as the land cools faster than the sea and air flows from land to sea; it is known as the land breeze (Mathew, 2006).

The weather and climate of the study areas are of the nature that the areas are being affected by wind shear occurrences. Murtala Mohammed International Airport in Lagos State and Port Harcourt International Airport in Rivers State are very close to the Atlantic Ocean, and wind shear is heavily triggered by the south-western trade wind from the ocean. It is therefore very pertinent that this study is carried out in the study areas.

\subsection{Study Area}

Murtala Mohammed International Airport, Lagos State and Port Harcourt International Airport, Rivers State are the two study areas of this study. Lagos State is located on the south-western part of Nigeria on the narrow coastal flood plain of the Bight of Benin. It lies approximately between latitude $6^{0} 22^{1} \mathrm{~N}$ and $6^{0} 42^{1} \mathrm{~N}$ of the equator and between longitude $2^{0} 42^{1}$ Eand $3^{0} 22^{1} \mathrm{E}$ of the Greenwich Meridian. It is bounded to the North andEast by Ogun State of Nigeria, to the West by the Republic of Benin, and to the South by the Atlantic Ocean. Territorially, Lagos State encompasses an area of 358,862 hectares or 3,577 km² (Building Nigeria's Response to Climate Change Project (BNRCC), 2012).

The population of Lagos State by the 2006 National Census conducted by the National Population Commission was $17,552,942$. Going by a population growth rate of 3.2 percent, the projected population for the State in 2015 is 23,305,971 (National Bureau of Statistics of Nigeria, 2013). Development in Lagos State is so rapid that the metropolitan area has expanded and absorbed the once rural communities. Hence, Lagos can best be described as a megacity. A megacity by definition is a continuous urbanized area with population of at least 10 million people(United Nations Centre for Human Settlements (UNCHS-Habitat), 1996). The population of the State has been above 10 million people since the 2006 National census. Some four Local Government Areas of Ogun State, adjoining Lagos State have fused into Lagos State forming Lagos Megacity Region(LMCR) (Oteri \& Ayeni, 2016).

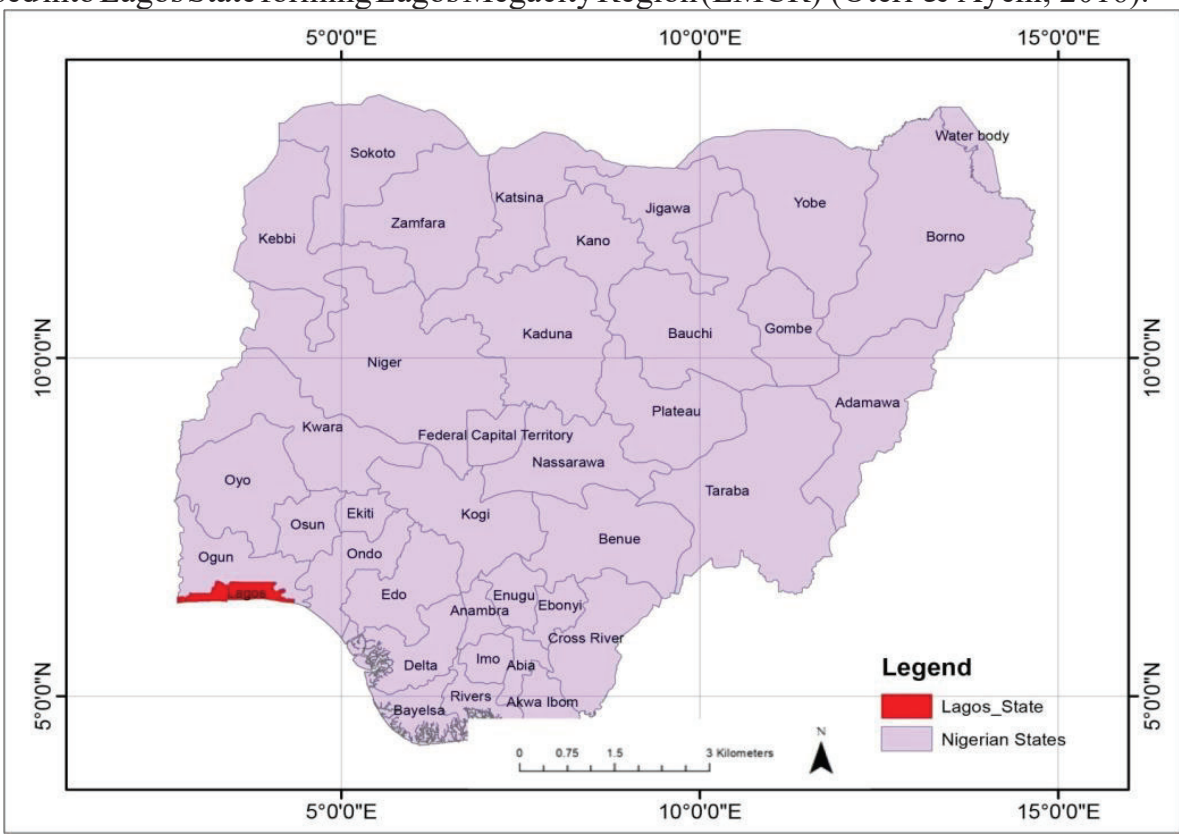

Fig. 1.1: Map of Nigeria showing Lagos State 


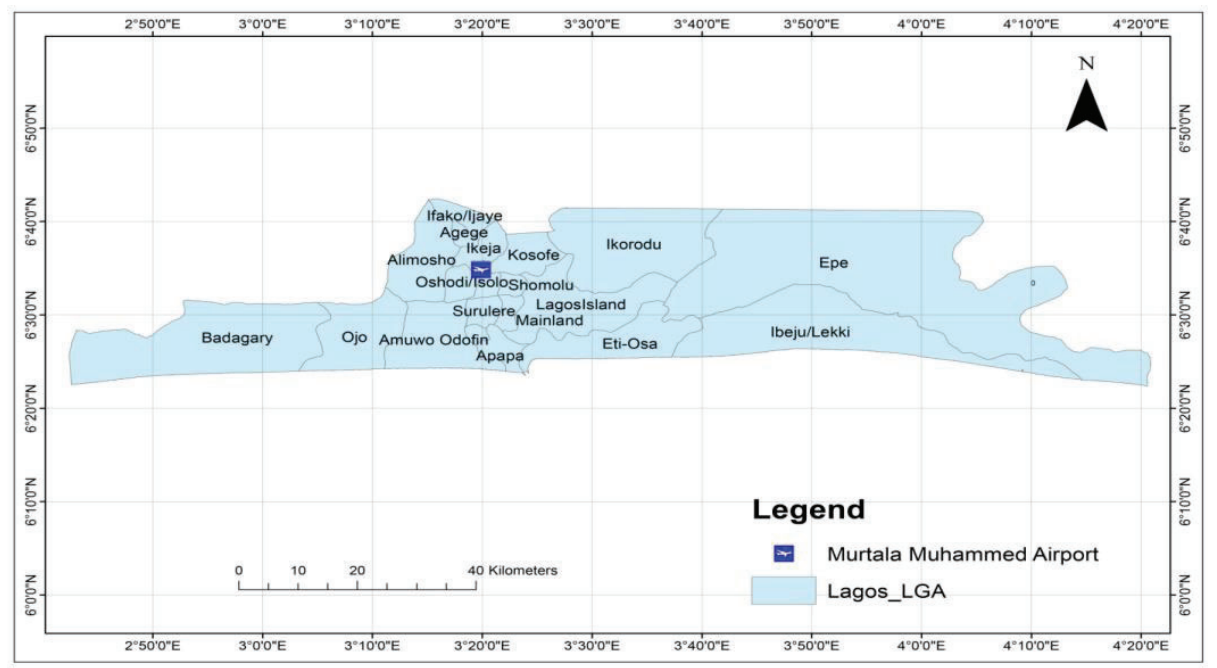

Fig. 1.2: Map of Lagos State showing Murtala Mohammed International Airport

Lagos State has a coastline of $180 \mathrm{~km}$ long (Oteri, 2013). Underlain by sedimentary rocks; it is on a coastal plain characterized by predominantly flat terrain, with an average elevation of less than $15 \mathrm{~m}$ above sea level. The land slopes gently from the interior to the sea. Water bodies and wetlands cover over 40 percent of the total land area of the State with lagoons and creeks consisting 22 percent of its area. An additional 12 percent is subject to seasonal flooding (Building Nigeria's Response to Climate Change Project (BNRCC), 2012). Lagos has a tropical wet and dry climate. It experiences two rainy seasons, with the heaviest rains falling from April to July and a weaker rainy season from September to November. There is a brief relatively dry spell in August and a longer dry season from December to March (Oteri \& Ayeni, 2016). Rainfall varies from one location to the other in Lagos Megacity. With its high mean annual rainfall, Lagos Megacity has abundant water resources in the form of surface water (rivers, lagoons, lakes and creeks) and groundwater. The major rivers are Ogun, Yewa, Aye, Owo, Oworu and Osun (Oteri \& Ayeni, 2016).

The dominant vegetation of the State is the tropical swamp forest consisting of fresh water and mangrove swamp forests both of which are influenced by the double rainfall pattern of the State, which makes the environment a wetland region, hence, the reference to Lagos as an environment of aquatic splendour. Its wetland environment is characterized by rich alluvial and terrallitic red-yellow soil, on which would be found dense luxuriant undergrowth, climbers, epiphytes and tropical hard woods (Oteri \& Ayeni, 2016).

Murtala Mohammed International Airport (MMIA) (IATA: LOS, ICAO: DNMM) is an international airport located in Ikeja, Lagos State, Nigeria, and is the major airport serving the entire country. The airport was initially built during World War II. Originally known as Lagos International Airport, it was renamed in the mid 1970s, during construction of the new international terminal, after a former and fourth Nigerian military head of state, Murtala Mohammed.

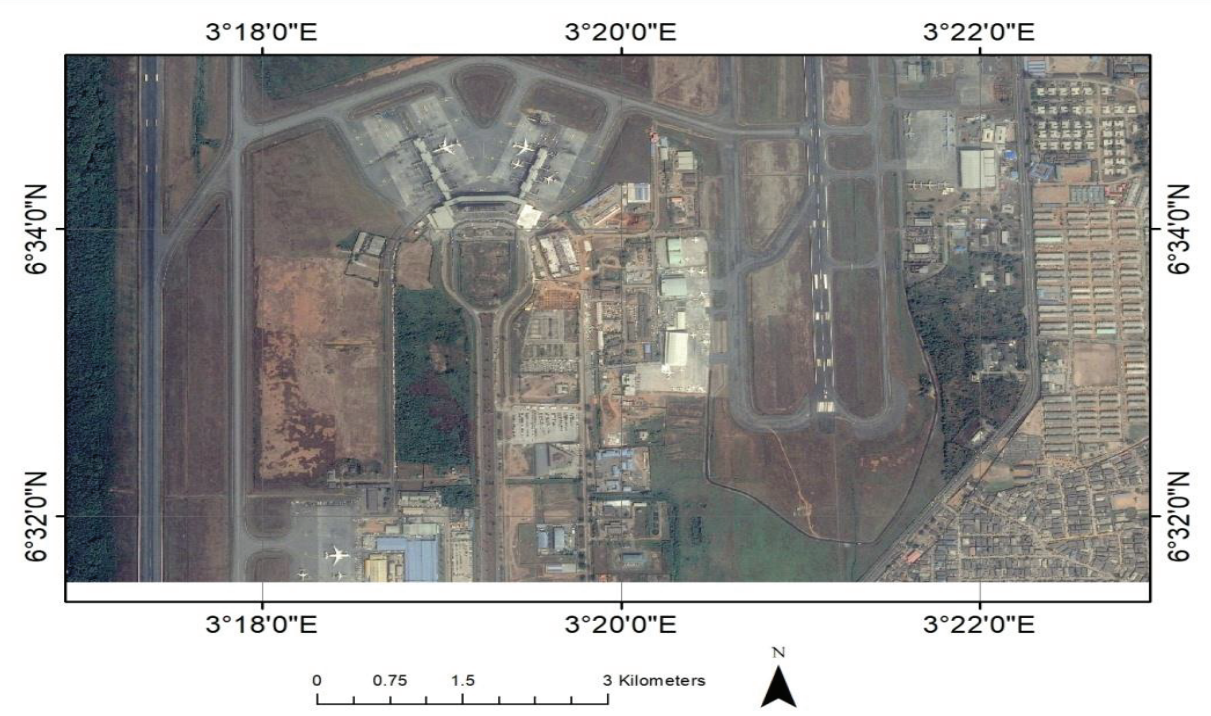

Fig. 1.3: Satellite Image of Murtala Mohammed International Airport 
Port Harcourt is situated at the southernmost part of Nigeria and it is located between latitude $4^{0} 30^{1}$ and $4^{0} 47^{1}$ north of the Equator and longitude $7^{0} 00^{1}$ and $7^{0} 15^{1}$ east of the Greenwich Meridian (Ajie \& Dienye, 2014). It is the largest city of Rivers State, Nigeria (The Tide News, 2013). It lies along the Bonny River and is located in Niger Delta (The Tide News, 2013). Port Harcourt is bounded to the Eastern and Western parts by meandering creeks and to the southern part by the first dockyard creek (Bonny River) and mangrove swamps. Towards the north where there is availability of land, it is bounded by Ikwerre Local Government Area (Ajie \& Dienye, 2014).

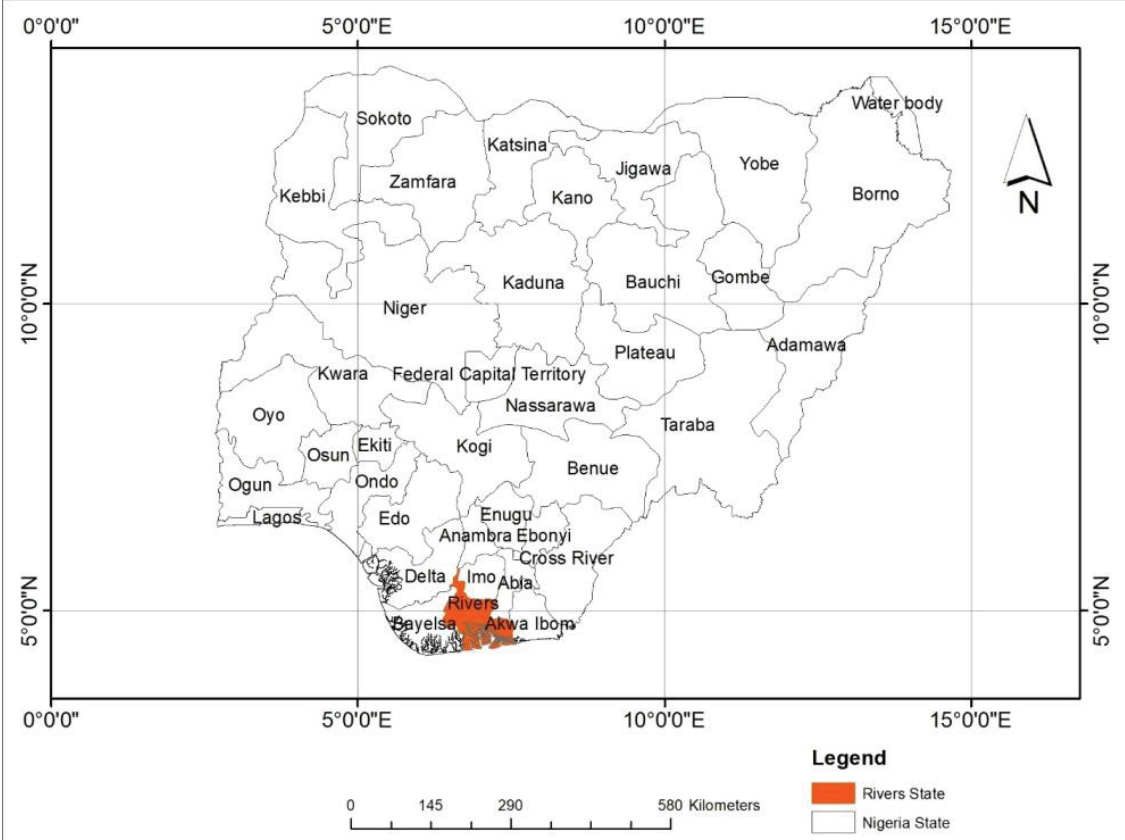

Fig. 1.4: Map of Nigeria showing Rivers State

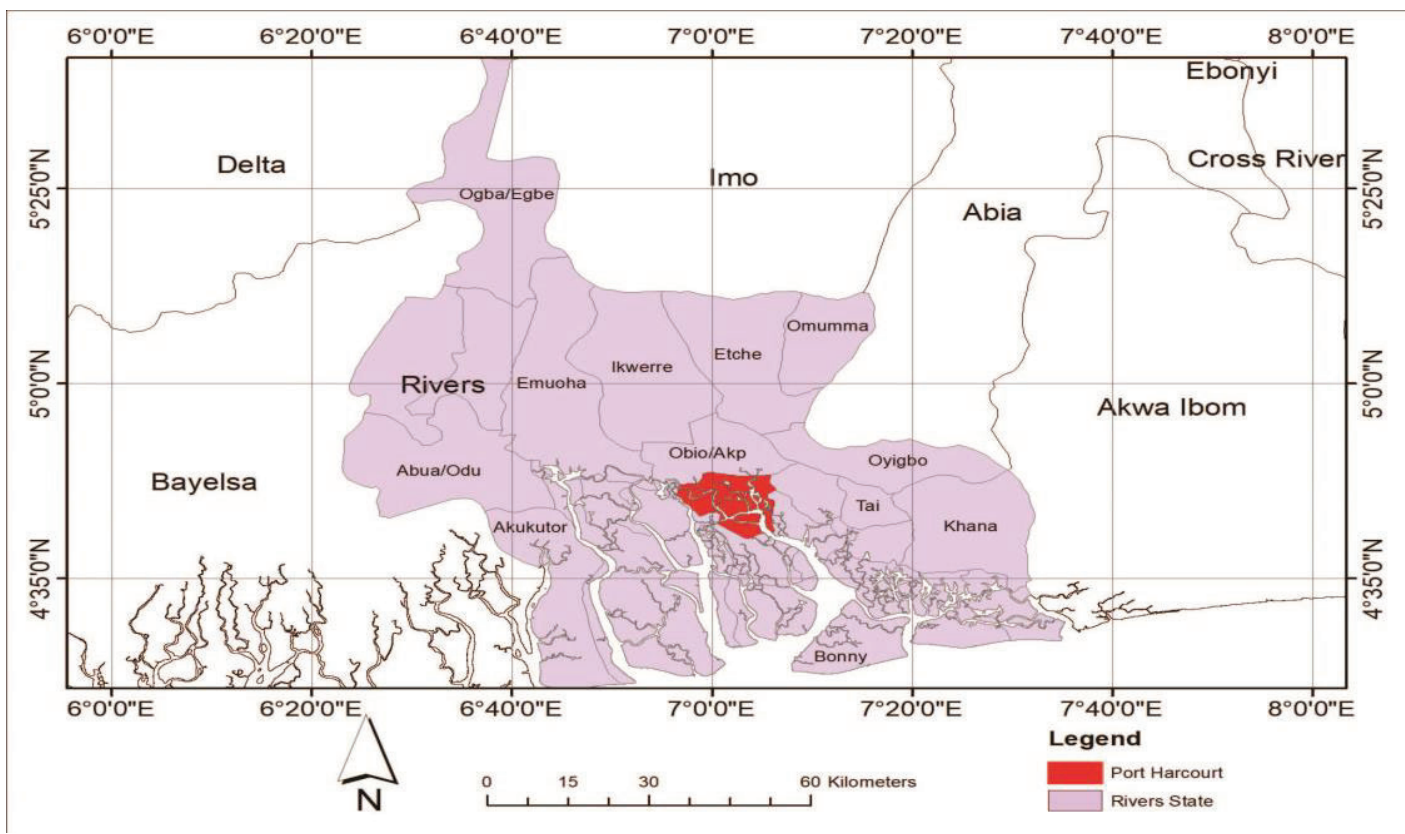

Fig. 1.5: Map of Rivers State showing Port Harcourt 


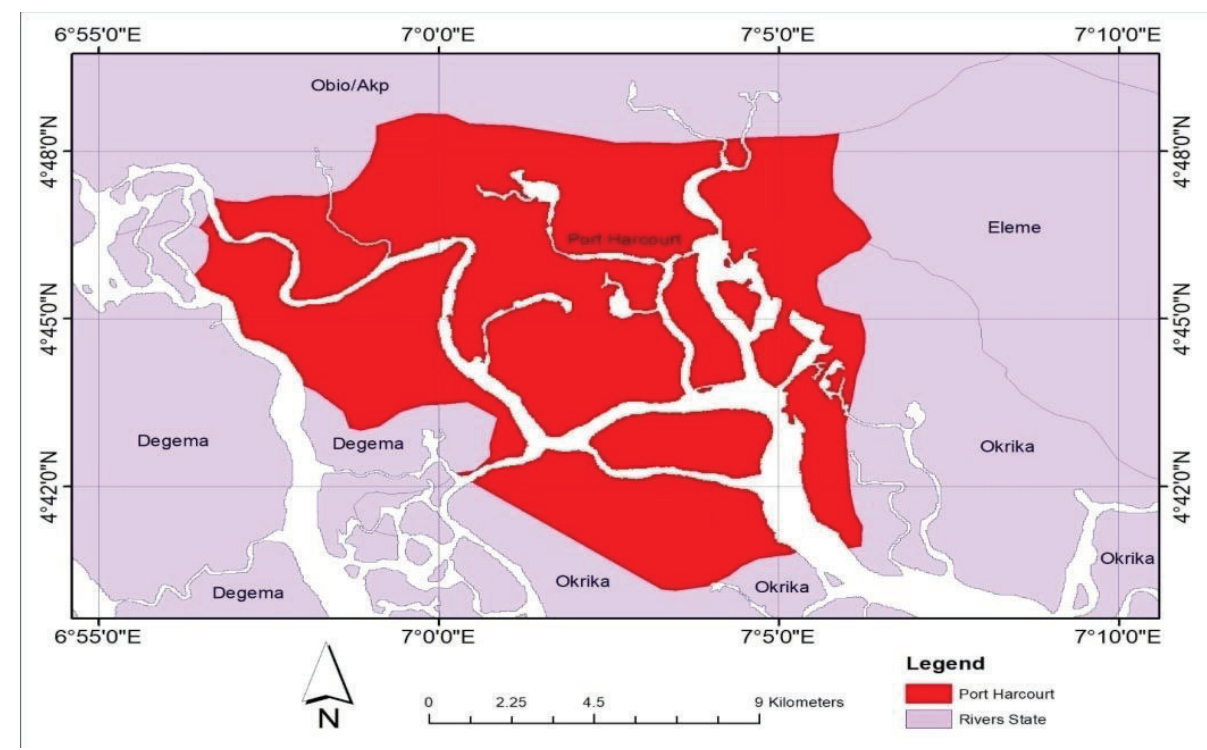

Fig. 1.6: Map of Port Harcourt

As of 2016, the Port Harcourt urban area has an estimated population of 1,865,000 inhabitants, up from 1,382,592 as of 2006 (Demographia, 2016). The ground surface of the area slopes from the north towards the Atlantic Ocean in the south (Nwankwoala \& Walter, 2012). Geologically, Port Harcourt as well as the entire Rivers State, lies within the Niger Delta Sedimentary Basin (Nwankwoala, Abam, Ede, Teme, \& Udom, 2008; Nwankwoala \& Walter, 2012). Lithostratigraphically, these rocks are divided into the Oldest Akata Formation (Paleocene), the Agbada Formation (Eocene) and the Youngest Benin Formation (Miocene) (Reyment, 1965; Short \& Stauble, 1967; Murat, 1970; Merki, 1970; Nwankwoala \& Walter, 2012).

The Port Harcourt Airport features a tropical monsoon climate with lengthy and heavy rainy seasons and very short dry seasons (Enete et al., 2015; World Meteorological Organization (WMO), 2018; National Oceanic and Atmospheric Administration (NOAA), 2018). Only the months of December and January truly qualifies as dry season months in the city (WMO, 2018; NOAA, 2018). The harmattan, which climatically influences many cities in West Africa, is less pronounced in Port Harcourt. Port Harcourt's heaviest precipitation occurs during September with an average of $369 \mathrm{~mm}$ of rain (WMO, 2018). Rainfall is seasonal, variable and energetic (Enete et al., 2015). Generally, south of latitude $05^{\circ} \mathrm{N}$, rain occurs on the average every month of the year but with varying duration circulation over the area (Enete et al., 2015). December on average is the driest month of the year; with an average rainfall of $20 \mathrm{~mm}$ dry seasons (Enete et al., 2015; WMO, 2018; NOAA, 2018). Temperatures throughout the year in the city are relatively constant, showing little variation throughout the course of the year. Average temperatures are typically between $25^{\circ} \mathrm{C}-28^{\circ} \mathrm{C}$ in the city (Enete et al., 2015; WMO, 2018; NOAA, 2018).

The high rainfall and humidity promotes thick vegetation termed tropical rainforest (Iloeje, 1979). The area also has both mangrove swamp forest and fresh water swamp forest. Mangrove swamp forest is permanently occupied by salt and tidal waters whereas fresh water swamp forests are seen in areas where fresh water dominates (Ezenwaji \& Chima, 2016).

The Port Harcourt International Airport lies between latitude $4^{\mathrm{o}} 72^{1} \mathrm{~N}$ and $4^{\mathrm{o}} 91^{1} \mathrm{~N}$ of the equator and longitude $6^{\mathrm{o}} 88^{1} \mathrm{E}$ and $7^{\mathrm{o}} 12^{1} \mathrm{E}$ of the Greenwich Meridian (Weli \& Emenike, 2016). 


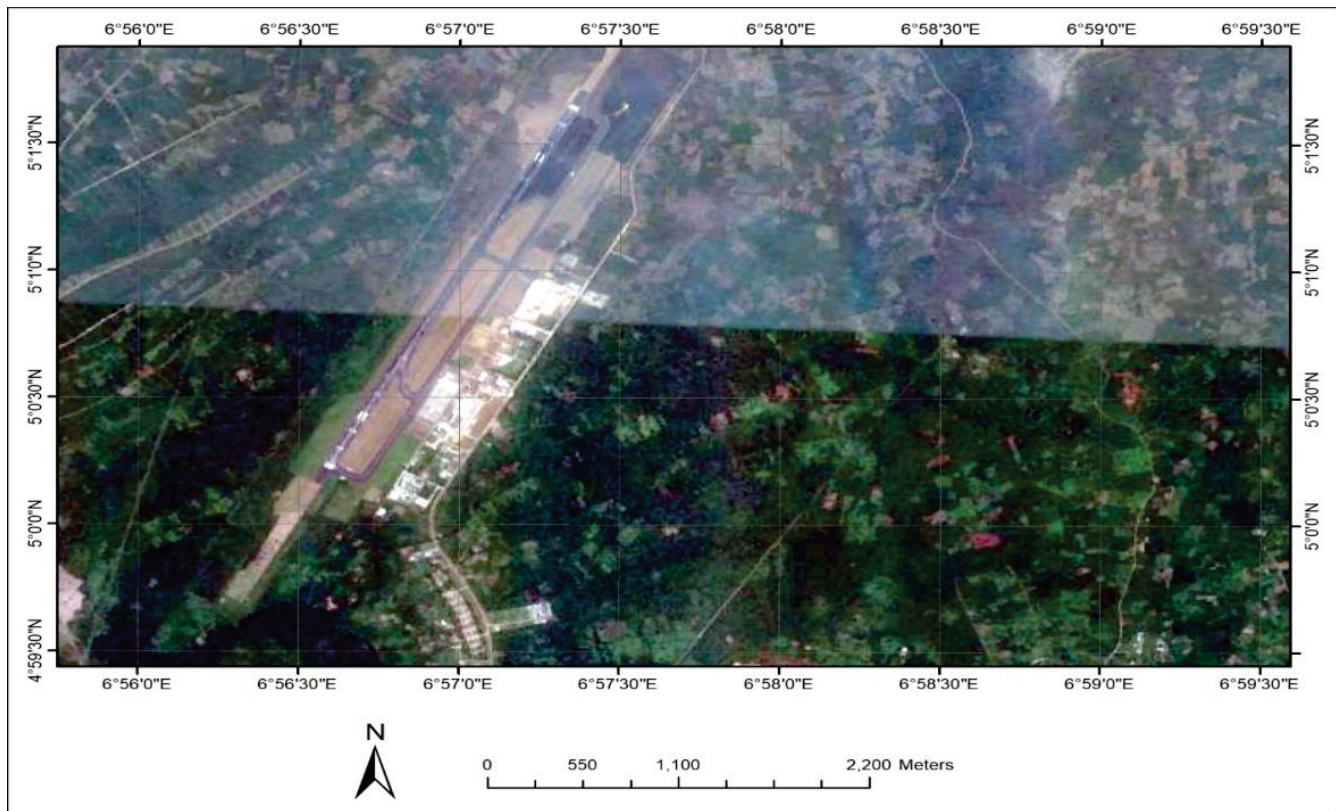

Fig. 1.7: Satellite Image of Port Harcourt International Airport

Port Harcourt International Airport (IATA:PHC,ICAO: DNPO) is an international airport located in Omagwa, a suburb of Port Harcourt city in Rivers State, Nigeria (Enete et al., 2015).

\section{Materials and Methods}

\subsection{Data Collection}

The annual time series secondary data of flight operations and wind shear used for this study were sourced from Nigerian Airspace Management Agency (NAMA) at Murtala Mohammed and Port Harcourt International Airports and Nigerian Meteorological Agency (NIMET) respectively. The data were compiled and presented in tables and charts for easy understanding and further analysis.

\subsection{Data Analysis}

\subsubsection{Calculation of wind shear at $50 \mathrm{~m}$ and $100 \mathrm{~m}$ above ground level (AGL)}

\subsubsection{Power Law}

The power law is a very simple and appropriate mathematical function that evaluates the wind shear at different heights (Wagner, Courtney, Gottschall, \& Marsden, 2011). It is a simple but useful model of the vertical wind profile which was first proposed by Hellman in 1916, derived empirically and that represents atmospheric wind profiles under atmospheric conditions where stability is not neutral. According to the power law, the wind profile is a function of thermal stability and surface roughness. It is used to evaluate increase in wind shear along with the increase in height. Equation 3.1 presents the formula for power law.

$\frac{V_{2}}{V_{1}}=\left(\frac{Z_{2}}{Z_{1}}\right)^{\propto}$

Where $V_{1}$ is the wind shear measured at certain height $Z_{1}, V_{2}$ is the wind shear measured at certain height $Z_{2}$, $\alpha$ is the wind shear exponent obtained empirically.

$$
\propto=\frac{\operatorname{In}\left(V_{2} / V_{1}\right)}{\operatorname{In}\left(Z_{2} / Z_{1}\right)}
$$

The wind shear exponent $(\alpha)$ is not constant and depends on numerous factors, including atmospheric conditions, time of day, season, wind speed, nature of terrain, temperature, mechanical mixing parameters, and wind shear exponent can also differ by measurement heights (Abdulla, 2014).

The statistical analysis techniques employed in achieving the research target are descriptive statistics which include such as mean, standard deviations, coefficient of variation and charts. The descriptive statistical techniques were employed in describing the behaviour of the data series over the period. Particularly, the mean and standard deviation captured the centre and spread of the dataset, while the charts pictured the annual and monthly trends of the data series together with the comparative evidences. Analytical packages used were Microsoft Excel, Eviews and Statistical Package for Social Sciences (SPSS) version 25.0 for windows. 
3 Results and Discussion

Table 3.1: Monthly distribution of Flight Diversions, Delays and Cancellation at Murtala Mohammed International Airport, Lagos (2008-2018)

\begin{tabular}{lccc}
\hline Months & No. of Flight Diversions & No. of Flight Delays & No. of Flight Cancellations \\
\hline Jan. & 29 & 19 & 14 \\
Feb. & 22 & 28 & 21 \\
Mar. & 31 & 57 & 33 \\
Apr & 29 & 57 & 30 \\
May & 33 & 73 & 35 \\
June & 42 & 66 & 29 \\
July & 47 & 59 & 34 \\
Aug. & 38 & 37 & 26 \\
Sept. & 26 & 63 & 39 \\
Oct. & 17 & 77 & 38 \\
Nov. & 30 & 31 & 23 \\
Dec. & 23 & 17 & 18 \\
\hline SUM & $\mathbf{3 6 7}$ & $\mathbf{5 8 4}$ & $\mathbf{3 4 0}$ \\
AVE. \pm Std. & $\mathbf{3 0 . 5 8} \pm \mathbf{8 . 5 4}$ & $\mathbf{4 8 . 6 7} \pm \mathbf{2 1 . 1 2}$ & $\mathbf{2 8 . 3 3} \pm \mathbf{8 . 0 3}$ \\
\hline Source Niger
\end{tabular}

Source: Nigerian Airspace Management Agency (NAMA), Lagos.

Table 3.2: Monthly distribution of Flight Diversions, Delays and Cancellation at Port Harcourt International Airport (2008-2018)

\begin{tabular}{cccc}
\hline Months & No. of Flight Diversions & No. of Flight Delays & No. of Flight Cancellations \\
\hline Jan. & 22 & 21 & 11 \\
Feb. & 17 & 33 & 17 \\
Mar. & 32 & 52 & 21 \\
Apr & 25 & 55 & 33 \\
May & 38 & 74 & 29 \\
June & 39 & 61 & 28 \\
July & 44 & 57 & 31 \\
Aug. & 33 & 30 & 21 \\
Sept. & 21 & 59 & 37 \\
Oct. & 19 & 75 & 35 \\
Nov. & 22 & 29 & 20 \\
Dec. & 20 & 18 & 15 \\
\hline SUM & $\mathbf{3 3 2}$ & $\mathbf{5 6 4}$ & $\mathbf{2 9 8}$ \\
AVE. \pm Std. & $\mathbf{2 7 . 6 7 \pm 9 . 1 1}$ & $\mathbf{4 7 \pm 1 9 . 9 3}$ & $\mathbf{2 4 . 8 3} \pm \mathbf{8 . 4 5}$ \\
\hline
\end{tabular}

Source: Nigerian Airspace Management Agency (NAMA), Port-Harcourt.

Table 3.3: Monthly distribution of wind shear measured at $20 \mathrm{~m}$ above ground level at Murtala Mohammed International Airport, Lagos (2008-2018)

\begin{tabular}{lcccccccccccc}
\hline M/Y & $\mathbf{2 0 0 8}$ & $\mathbf{2 0 0 9}$ & $\mathbf{2 0 1 0}$ & $\mathbf{2 0 1 1}$ & $\mathbf{2 0 1 2}$ & $\mathbf{2 0 1 3}$ & $\mathbf{2 0 1 4}$ & $\mathbf{2 0 1 5}$ & $\mathbf{2 0 1 6}$ & $\mathbf{2 0 1 7}$ & $\mathbf{2 0 1 8}$ & Ave. \\
\hline JAN & 7.2 & 4.4 & 3.0 & 2.7 & 3.5 & 3.6 & 4.7 & 4.2 & 3.0 & 4.0 & 3.9 & 4.0 \\
FEB & 8.7 & 8.7 & 3.0 & 4.4 & 3.8 & 5.0 & 4.0 & 4.3 & 3.0 & 3.8 & 4.0 & 4.8 \\
MAR & 8.4 & 9.0 & 4.4 & 4.7 & 3.7 & 4.6 & 5.0 & 4.0 & 3.6 & 4.2 & 4.3 & 5.1 \\
APR & 9.0 & 9.8 & 5.0 & 4.5 & 4.2 & 4.8 & 1.2 & 4.0 & 5.0 & 3.4 & 3.7 & 5.0 \\
MAY & 6.1 & 9.8 & 4.0 & 3.9 & 3.6 & 2.7 & 4.0 & 4.0 & 2.9 & 15.6 & 13.0 & 6.3 \\
JUN & 5.9 & 7.7 & 3.4 & 3.7 & 3.0 & 4.3 & 3.9 & 5.0 & 3.0 & 4.0 & 4.0 & 4.4 \\
JUL & 8.7 & 9.8 & 3.6 & 6.0 & 3.8 & 5.7 & 4.8 & 4.2 & 3.1 & 4.0 & 4.4 & 5.3 \\
AUG & 9.7 & 10.5 & 4.6 & 7.5 & 5.4 & 8.0 & 5.2 & 4.7 & 3.0 & 4.3 & 5.0 & 6.2 \\
SEP & 8.0 & 7.6 & 4.0 & 7.3 & 6.0 & 7.0 & 4.0 & 4.0 & 2.0 & 3.3 & 4.1 & 5.2 \\
OCT & 5.5 & 5.7 & 2.7 & 2.6 & 3.9 & 3.6 & 3.4 & 3.0 & 2.0 & 2.8 & 3.0 & 3.5 \\
NOV & 4.1 & 5.9 & 3.1 & 2.6 & 3.5 & 3.7 & 3.5 & 2.7 & 2.0 & 2.7 & 2.9 & 3.3 \\
DEC & 5.0 & 5.0 & 3.2 & 5.1 & 3.1 & 3.9 & 3.7 & 4.0 & 2.3 & 3.3 & 3.4 & 3.8 \\
\hline SUM & $\mathbf{8 6 . 3}$ & $\mathbf{9 3 . 9}$ & $\mathbf{4 4}$ & $\mathbf{5 5}$ & $\mathbf{4 7 . 5}$ & $\mathbf{5 6 . 9}$ & $\mathbf{4 7 . 4}$ & $\mathbf{4 8 . 1}$ & $\mathbf{3 4 . 9}$ & $\mathbf{5 5 . 4}$ & $\mathbf{5 5 . 7}$ & $\mathbf{5 6 . 9}$ \\
AVE. & $\mathbf{7 . 1 9}$ & $\mathbf{7 . 8 3}$ & $\mathbf{3 . 6 7}$ & $\mathbf{4 . 5 8}$ & $\mathbf{3 . 9 6}$ & $\mathbf{4 . 7 4}$ & $\mathbf{3 . 9 5}$ & $\mathbf{4 . 0 1}$ & $\mathbf{2 . 9 1}$ & $\mathbf{4 . 6 2}$ & $\mathbf{4 . 6 4}$ & $\mathbf{4 . 7 4}$ \\
Std. & $\mathbf{1 . 8 2}$ & $\mathbf{2 . 1 1}$ & $\mathbf{0 . 7 3}$ & $\mathbf{1 . 6 8}$ & $\mathbf{0 . 8 9}$ & $\mathbf{1 . 5 2}$ & $\mathbf{1 . 0 5}$ & $\mathbf{0 . 6 3}$ & $\mathbf{0 . 8 4}$ & $\mathbf{3 . 5 0}$ & $\mathbf{2 . 7 0}$ & $\mathbf{0 . 9 8}$ \\
\hline Source & NIMET & & & & & & & & & & &
\end{tabular}

Source: NIMET 
Table 3.4: Monthly distribution of wind shear measured at 20m above ground level at Port Harcourt International Airport (2008-2018)

\begin{tabular}{lcccccccccccc}
\hline M/Y & $\mathbf{2 0 0 8}$ & $\mathbf{2 0 0 9}$ & $\mathbf{2 0 1 0}$ & $\mathbf{2 0 1 1}$ & $\mathbf{2 0 1 2}$ & $\mathbf{2 0 1 3}$ & $\mathbf{2 0 1 4}$ & $\mathbf{2 0 1 5}$ & $\mathbf{2 0 1 6}$ & $\mathbf{2 0 1 7}$ & $\mathbf{2 0 1 8}$ & Ave. \\
\hline JAN & 3.3 & 5.3 & 4.0 & 3.8 & 2.4 & 2.4 & 2.4 & 3.0 & 2.4 & 2.1 & 2.0 & 3.0 \\
FEB & 3.8 & 2.3 & 4.0 & 2.8 & 2.4 & 2.5 & 3.2 & 3.1 & 3.2 & 2.4 & 3.0 & 3.0 \\
MAR & 3.2 & 2.3 & 4.0 & 2.9 & 2.5 & 2.5 & 2.7 & 2.4 & 2.7 & 2.7 & 2.1 & 2.7 \\
APR & 2.4 & 2.6 & 4.0 & 2.2 & 2.4 & 2.3 & 2.7 & 2.4 & 2.7 & 3.0 & 2.8 & 2.7 \\
MAY & 2.3 & 2.3 & 4.0 & 1.7 & 2.4 & 2.4 & 2.7 & 2.6 & 2.7 & 2.1 & 2.5 & 2.5 \\
JUN & 2.2 & 2.6 & 3.0 & 2.2 & 2.7 & 2.3 & 2.8 & 2.9 & 2.7 & 2.3 & 2.4 & 2.6 \\
JUL & 2.2 & 3.5 & 2.0 & 2.7 & 2.4 & 2.5 & 2.5 & 2.4 & 2.4 & 2.6 & 2.5 & 2.5 \\
AUG & 2.4 & 2.1 & 3.0 & 3.5 & 2.8 & 2.7 & 2.7 & 2.8 & 2.7 & 3.1 & 2.8 & 2.8 \\
SEP & 1.9 & 2.3 & 4.0 & 2.3 & 2.4 & 2.7 & 2.5 & 2.7 & 2.6 & 2.8 & 2.7 & 2.6 \\
OCT & 2.1 & 2.8 & 3.0 & 2.2 & 2.2 & 2.3 & 2.1 & 2.0 & 2.2 & 2.0 & 2.0 & 2.3 \\
NOV & 1.6 & 2.2 & 3.0 & 1.8 & 1.9 & 1.9 & 1.9 & 1.9 & 2.0 & 2.0 & 1.7 & 2.0 \\
DEC & 1.5 & 1.8 & 3.0 & 3.1 & 1.9 & 2.1 & 1.8 & 2.6 & 1.8 & 2.0 & 2.0 & 2.1 \\
\hline SUM & $\mathbf{2 8 . 9}$ & $\mathbf{3 2 . 1}$ & $\mathbf{4 1}$ & $\mathbf{3 1 . 2}$ & $\mathbf{2 8 . 4}$ & $\mathbf{2 8 . 6}$ & $\mathbf{3 0}$ & $\mathbf{3 0 . 8}$ & $\mathbf{3 0 . 1}$ & $\mathbf{2 9 . 1}$ & $\mathbf{2 8 . 5}$ & $\mathbf{3 0 . 8}$ \\
AVE. & $\mathbf{2 . 4 1}$ & $\mathbf{2 . 6 8}$ & $\mathbf{3 . 4 2}$ & $\mathbf{2 . 6 0}$ & $\mathbf{2 . 3 7}$ & $\mathbf{2 . 3 8}$ & $\mathbf{2 . 5 0}$ & $\mathbf{2 . 5 7}$ & $\mathbf{2 . 5 1}$ & $\mathbf{2 . 4 3}$ & $\mathbf{2 . 3 8}$ & $\mathbf{2 . 5 7}$ \\
Std. & $\mathbf{0 . 6 9}$ & $\mathbf{0 . 9 3}$ & $\mathbf{0 . 6 7}$ & $\mathbf{0 . 6 5}$ & $\mathbf{0 . 2 7}$ & $\mathbf{0 . 2 3}$ & $\mathbf{0 . 4 0}$ & $\mathbf{0 . 3 7}$ & $\mathbf{0 . 3 8}$ & $\mathbf{0 . 4 1}$ & $\mathbf{0 . 4 1}$ & $\mathbf{0 . 3 1}$ \\
\hline
\end{tabular}

Source: NIMET

Table 3.5: Monthly distribution of wind shear extrapolated at $50 \mathrm{~m}$ above ground level at Murtala Mohammed International Airport, Lagos (2008-2018)

\begin{tabular}{|c|c|c|c|c|c|c|c|c|c|c|c|c|}
\hline $\mathbf{M} / \mathbf{Y}$ & 2008 & 2009 & 2010 & 2011 & 2012 & 2013 & 2014 & 2015 & 2016 & 2017 & 2018 & Ave. \\
\hline Jan. & 8.2 & 5.0 & 3.4 & 3.1 & 4.0 & 4.1 & 5.4 & 4.8 & 3.4 & 4.6 & 4.4 & 4.6 \\
\hline Feb. & 9.9 & 9.9 & 3.4 & 5.0 & 4.3 & 5.7 & 4.6 & 4.9 & 3.4 & 4.3 & 4.6 & 5.5 \\
\hline Mar. & 9.6 & 10.3 & 5.0 & 5.4 & 4.2 & 5.2 & 5.7 & 4.6 & 4.1 & 4.8 & 4.9 & 5.8 \\
\hline Apr. & 10.3 & 11.2 & 5.7 & 5.1 & 4.8 & 5.5 & 1.4 & 4.6 & 5.7 & 3.9 & 4.2 & 5.7 \\
\hline May & 7.0 & 11.2 & 4.6 & 4.4 & 4.1 & 3.1 & 4.6 & 4.6 & 3.3 & 17.8 & 14.8 & 7.2 \\
\hline Jun. & 6.7 & 8.8 & 3.9 & 4.2 & 3.4 & 4.9 & 4.4 & 5.7 & 3.4 & 4.6 & 4.6 & 5.0 \\
\hline Jul. & 9.9 & 11.2 & 4.1 & 6.8 & 4.3 & 6.5 & 5.5 & 4.8 & 3.5 & 4.6 & 5.0 & 6.0 \\
\hline Aug. & 11.1 & 12.0 & 5.2 & 8.6 & 6.2 & 9.1 & 6.0 & 5.4 & 3.4 & 4.9 & 5.7 & 7.1 \\
\hline Sep. & 9.1 & 8.7 & 4.6 & 8.3 & 6.8 & 8.0 & 4.6 & 4.6 & 2.3 & 3.8 & 4.7 & 6.0 \\
\hline Oct. & 6.3 & 6.5 & 3.1 & 3.0 & 4.4 & 4.1 & 3.9 & 3.4 & 2.3 & 3.2 & 3.4 & 4.0 \\
\hline Nov. & 4.7 & 6.7 & 3.5 & 3.0 & 4.0 & 4.2 & 4.0 & 3.1 & 2.3 & 3.1 & 3.3 & 3.8 \\
\hline Dec. & 5.7 & 5.7 & 3.6 & 5.8 & 3.5 & 4.4 & 4.2 & 4.6 & 2.6 & 3.8 & 3.9 & 4.3 \\
\hline SUM & 98.5 & 107.2 & 50.1 & 62.7 & 54 & 64.8 & 54.3 & 55.1 & 39.7 & 63.4 & 63.5 & 64.8 \\
\hline AVE. & 8.21 & 8.93 & 4.18 & 5.23 & 4.50 & 5.40 & 4.53 & 4.59 & 3.31 & 5.28 & 5.29 & 5.4 \\
\hline Std. & 2.07 & 2.42 & 0.83 & 1.91 & 1.01 & 1.74 & 1.20 & 0.72 & 0.95 & 3.99 & 3.07 & 1.11 \\
\hline
\end{tabular}

Source: Authors' Computation

Table 3.6: Monthly distribution of wind shear extrapolated at 50m above ground level at Port Harcourt International Airport (2008-2018)

\begin{tabular}{lcccccccccccc}
\hline M/Y & $\mathbf{2 0 0 8}$ & $\mathbf{2 0 0 9}$ & $\mathbf{2 0 1 0}$ & $\mathbf{2 0 1 1}$ & $\mathbf{2 0 1 2}$ & $\mathbf{2 0 1 3}$ & $\mathbf{2 0 1 4}$ & $\mathbf{2 0 1 5}$ & $\mathbf{2 0 1 6}$ & $\mathbf{2 0 1 7}$ & $\mathbf{2 0 1 8}$ & Ave. \\
\hline Jan. & 3.7 & 6.0 & 4.6 & 4.3 & 2.7 & 2.7 & 2.7 & 3.4 & 2.7 & 2.4 & 2.3 & 3.4 \\
Feb. & 4.3 & 2.6 & 4.6 & 3.2 & 2.7 & 2.9 & 3.6 & 3.5 & 3.6 & 2.7 & 3.4 & 3.4 \\
Mar. & 3.6 & 2.6 & 4.6 & 3.3 & 2.9 & 2.9 & 3.1 & 2.7 & 3.1 & 3.1 & 2.4 & 3.1 \\
Apr. & 2.7 & 3.0 & 4.6 & 2.5 & 2.7 & 2.6 & 3.1 & 2.7 & 3.1 & 3.4 & 3.2 & 3.1 \\
May & 2.6 & 2.6 & 4.6 & 1.9 & 2.7 & 2.7 & 3.1 & 3.0 & 3.1 & 2.4 & 2.9 & 2.9 \\
Jun. & 2.5 & 3.0 & 3.4 & 2.5 & 3.1 & 2.6 & 3.2 & 3.3 & 3.1 & 2.6 & 2.7 & 2.9 \\
Jul. & 2.5 & 4.0 & 2.3 & 3.1 & 2.7 & 2.9 & 2.9 & 2.7 & 2.7 & 3.0 & 2.9 & 2.9 \\
Aug. & 2.7 & 2.4 & 3.4 & 4.0 & 3.2 & 3.1 & 3.1 & 3.2 & 3.1 & 3.5 & 3.2 & 3.2 \\
Sep. & 2.2 & 2.6 & 4.6 & 2.6 & 2.4 & 3.1 & 2.9 & 3.1 & 3.0 & 3.2 & 3.1 & 3.0 \\
Oct. & 2.4 & 3.2 & 3.4 & 2.5 & 2.5 & 2.6 & 2.4 & 2.3 & 2.5 & 2.3 & 2.3 & 2.6 \\
Nov. & 1.8 & 2.5 & 3.4 & 2.1 & 2.2 & 2.2 & 2.2 & 2.2 & 2.3 & 2.3 & 1.9 & 2.3 \\
Dec. & 1.7 & 2.1 & 3.4 & 3.5 & 2.2 & 2.4 & 2.1 & 3.0 & 2.1 & 2.3 & 2.3 & 2.5 \\
\hline SUM & $\mathbf{3 2 . 7 0}$ & $\mathbf{3 6 . 6 0}$ & $\mathbf{4 6 . 9 0}$ & $\mathbf{3 5 . 5 0}$ & $\mathbf{3 2 . 0 0}$ & $\mathbf{3 2 . 7 0}$ & $\mathbf{3 4 . 4 0}$ & $\mathbf{3 5 . 1 0}$ & $\mathbf{3 4 . 4 0}$ & $\mathbf{3 3 . 2 0}$ & $\mathbf{3 2 . 6 0}$ & $\mathbf{3 5 . 3 0}$ \\
AVE. & $\mathbf{2 . 7 3}$ & $\mathbf{3 . 0 5}$ & $\mathbf{3 . 9 1}$ & $\mathbf{2 . 9 6}$ & $\mathbf{2 . 6 7}$ & $\mathbf{2 . 7 3}$ & $\mathbf{2 . 8 7}$ & $\mathbf{2 . 9 3}$ & $\mathbf{2 . 8 7}$ & $\mathbf{2 . 7 7}$ & $\mathbf{2 . 7 2}$ & $\mathbf{2 . 9 4}$ \\
Std. & $\mathbf{0 . 7 8}$ & $\mathbf{1 . 0 5}$ & $\mathbf{0 . 7 8}$ & $\mathbf{0 . 7 4}$ & $\mathbf{0 . 3 1}$ & $\mathbf{0 . 2 7}$ & $\mathbf{0 . 4 4}$ & $\mathbf{0 . 4 1}$ & $\mathbf{0 . 4 2}$ & $\mathbf{0 . 4 5}$ & $\mathbf{0 . 4 7}$ & $\mathbf{0 . 3 4}$ \\
\hline
\end{tabular}

Source: Authors' Computation 
Table 3.7: Monthly distribution of wind shear extrapolated at $100 \mathrm{~m}$ above ground level at Murtala Mohammed International Airport, Lagos (2008-2018)

\begin{tabular}{lcccccccccccc}
\hline M/Y & $\mathbf{2 0 0 8}$ & $\mathbf{2 0 0 9}$ & $\mathbf{2 0 1 0}$ & $\mathbf{2 0 1 1}$ & $\mathbf{2 0 1 2}$ & $\mathbf{2 0 1 3}$ & $\mathbf{2 0 1 4}$ & $\mathbf{2 0 1 5}$ & $\mathbf{2 0 1 6}$ & $\mathbf{2 0 1 7}$ & $\mathbf{2 0 1 8}$ & Ave. \\
\hline Jan. & 9.0 & 5.3 & 3.8 & 3.4 & 4.4 & 4.5 & 5.9 & 5.3 & 3.8 & 5.0 & 4.9 & 5.0 \\
Feb. & 10.9 & 10.9 & 3.8 & 5.5 & 4.8 & 6.3 & 5.0 & 5.4 & 3.8 & 4.8 & 5.0 & 6.0 \\
Mar. & 10.5 & 11.3 & 5.5 & 5.9 & 4.6 & 5.8 & 6.3 & 5.0 & 4.5 & 5.3 & 5.4 & 6.4 \\
Apr. & 11.3 & 12.3 & 6.3 & 5.6 & 5.3 & 6.0 & 1.5 & 5.0 & 6.3 & 4.3 & 4.6 & 6.2 \\
May & 7.6 & 12.3 & 5.0 & 4.9 & 4.5 & 3.4 & 5.0 & 5.0 & 3.6 & 19.5 & 16.3 & 7.9 \\
Jun. & 7.4 & 9.6 & 4.3 & 4.6 & 3.8 & 5.4 & 4.9 & 6.3 & 3.8 & 5.0 & 5.0 & 5.5 \\
Jul. & 10.9 & 12.3 & 4.5 & 7.5 & 4.8 & 7.1 & 6.0 & 5.3 & 3.9 & 5.0 & 5.5 & 6.6 \\
Aug. & 12.1 & 13.1 & 5.8 & 9.4 & 6.8 & 10.0 & 6.5 & 5.9 & 3.8 & 5.4 & 6.3 & 7.7 \\
Sep. & 10.0 & 9.5 & 5.0 & 9.1 & 7.5 & 8.8 & 5.0 & 5.0 & 2.5 & 4.1 & 5.1 & 6.5 \\
Oct. & 6.9 & 7.1 & 3.4 & 3.3 & 4.9 & 4.5 & 4.3 & 3.8 & 2.5 & 3.5 & 3.8 & 4.4 \\
Nov. & 5.1 & 7.4 & 3.9 & 3.3 & 4.4 & 4.6 & 4.4 & 3.4 & 2.5 & 3.4 & 3.6 & 4.2 \\
Dec. & 6.3 & 6.3 & 4.0 & 6.4 & 3.9 & 4.9 & 4.6 & 5.0 & 2.9 & 4.1 & 4.3 & 4.8 \\
\hline SUM & $\mathbf{1 0 8 . 0 0}$ & $\mathbf{1 1 7 . 4 0}$ & $\mathbf{5 5 . 3 0}$ & $\mathbf{6 8 . 9 0}$ & $\mathbf{5 9 . 7 0}$ & $\mathbf{7 1 . 3 0}$ & $\mathbf{5 9 . 4 0}$ & $\mathbf{6 0 . 4 0}$ & $\mathbf{4 3 . 9 0}$ & $\mathbf{6 9 . 4 0}$ & $\mathbf{6 9 . 8 0}$ & $\mathbf{7 1 . 2}$ \\
AVE. & $\mathbf{9 . 0 0}$ & $\mathbf{9 . 7 8}$ & $\mathbf{4 . 6 1}$ & $\mathbf{5 . 7 4}$ & $\mathbf{4 . 9 8}$ & $\mathbf{5 . 9 4}$ & $\mathbf{4 . 9 5}$ & $\mathbf{5 . 0 3}$ & $\mathbf{3 . 6 6}$ & $\mathbf{5 . 7 8}$ & $\mathbf{5 . 8 2}$ & $\mathbf{5 . 9 3}$ \\
Std. & $\mathbf{2 . 2 7}$ & $\mathbf{2 . 6 8}$ & $\mathbf{0 . 9 1}$ & $\mathbf{2 . 0 8}$ & $\mathbf{1 . 1 1}$ & $\mathbf{1 . 9 1}$ & $\mathbf{1 . 3 1}$ & $\mathbf{0 . 7 9}$ & $\mathbf{1 . 0 6}$ & $\mathbf{4 . 3 7}$ & $\mathbf{3 . 3 8}$ & $\mathbf{1 . 2 0}$ \\
\hline
\end{tabular}

Source: Authors' Computation

Table 3.8: Monthly distribution of wind shear extrapolated at $100 \mathrm{~m}$ above ground level at Port Harcourt International Airport (2008-2018)

\begin{tabular}{lcccccccccccc}
\hline M/Y & $\mathbf{2 0 0 8}$ & $\mathbf{2 0 0 9}$ & $\mathbf{2 0 1 0}$ & $\mathbf{2 0 1 1}$ & $\mathbf{2 0 1 2}$ & $\mathbf{2 0 1 3}$ & $\mathbf{2 0 1 4}$ & $\mathbf{2 0 1 5}$ & $\mathbf{2 0 1 6}$ & $\mathbf{2 0 1 7}$ & $\mathbf{2 0 1 8}$ & Ave. \\
\hline Jan. & 4.1 & 6.6 & 5.0 & 4.8 & 3.0 & 3.0 & 3.0 & 3.8 & 3.0 & 2.6 & 2.5 & 3.8 \\
Feb. & 4.8 & 2.9 & 5.0 & 3.5 & 3.0 & 3.1 & 4.0 & 3.9 & 4.0 & 3.0 & 3.8 & 3.7 \\
Mar. & 4.0 & 2.9 & 5.0 & 3.6 & 3.1 & 3.1 & 3.4 & 3.0 & 3.4 & 3.4 & 2.6 & 3.4 \\
Apr. & 3.0 & 3.3 & 5.0 & 2.8 & 3.0 & 2.9 & 3.4 & 3.0 & 3.4 & 3.8 & 3.5 & 3.4 \\
May & 2.9 & 2.9 & 5.0 & 2.1 & 3.0 & 3.0 & 3.4 & 3.3 & 3.4 & 2.6 & 3.1 & 3.2 \\
Jun. & 2.8 & 3.3 & 3.8 & 2.8 & 3.4 & 2.9 & 3.5 & 3.6 & 3.4 & 2.9 & 3.0 & 3.2 \\
Jul. & 2.8 & 4.4 & 2.5 & 3.4 & 3.0 & 3.1 & 3.1 & 3.0 & 3.0 & 3.3 & 3.1 & 3.2 \\
Aug. & 3.0 & 2.6 & 3.8 & 4.4 & 3.5 & 3.4 & 3.4 & 3.5 & 3.4 & 3.9 & 3.5 & 3.5 \\
Sep. & 2.4 & 2.9 & 5.0 & 2.9 & 3.0 & 3.4 & 3.1 & 3.4 & 3.3 & 3.5 & 3.4 & 3.3 \\
Oct. & 2.6 & 3.5 & 3.8 & 2.8 & 2.8 & 2.9 & 2.6 & 2.5 & 2.8 & 2.5 & 2.5 & 2.8 \\
Nov. & 2.0 & 2.8 & 3.8 & 2.3 & 2.4 & 2.4 & 2.4 & 2.4 & 2.5 & 2.5 & 2.1 & 2.5 \\
Dec. & 1.9 & 2.3 & 3.8 & 3.9 & 2.4 & 2.6 & 2.3 & 3.3 & 2.3 & 2.5 & 2.5 & 2.7 \\
\hline SUM & $\mathbf{3 6 . 3 0}$ & $\mathbf{4 0 . 4 0}$ & $\mathbf{5 1 . 5 0}$ & $\mathbf{3 9 . 3 0}$ & $\mathbf{3 5 . 6 0}$ & $\mathbf{3 5 . 8 0}$ & $\mathbf{3 7 . 6 0}$ & $\mathbf{3 8 . 7 0}$ & $\mathbf{3 7 . 9 0}$ & $\mathbf{3 6 . 5 0}$ & $\mathbf{3 5 . 6 0}$ & $\mathbf{3 8 . 7 0}$ \\
AVE. & $\mathbf{3 . 0 3}$ & $\mathbf{3 . 3 7}$ & $\mathbf{4 . 2 9}$ & $\mathbf{3 . 2 8}$ & $\mathbf{2 . 9 7}$ & $\mathbf{2 . 9 8}$ & $\mathbf{3 . 1 3}$ & $\mathbf{3 . 2 3}$ & $\mathbf{3 . 1 6}$ & $\mathbf{3 . 0 4}$ & $\mathbf{2 . 9 7}$ & $\mathbf{3 . 2 3}$ \\
Std. & $\mathbf{0 . 8 7}$ & $\mathbf{1 . 1 5}$ & $\mathbf{0 . 8 2}$ & $\mathbf{0 . 8 2}$ & $\mathbf{0 . 3 3}$ & $\mathbf{0 . 2 9}$ & $\mathbf{0 . 5 0}$ & $\mathbf{0 . 4 7}$ & $\mathbf{0 . 4 6}$ & $\mathbf{0 . 5 2}$ & $\mathbf{0 . 5 3}$ & $\mathbf{0 . 3 9}$ \\
\hline
\end{tabular}

Source: Authors' Computation

3.1 Extrapolating wind shear at 50 meters and 100 meters above ground level using power law model. The extrapolation of wind shear at $50 \mathrm{~m}$ and $100 \mathrm{~m}$ above ground level at Murtala Mohammed International Airport and Port Harcourt International Airport were done using the power law model (equation 2.1). From table 3.3 to 3.8 , it can be observed that the values of wind shear extrapolated at 50 meters and 100 meters above ground level at the airports are higher than the values of wind shear measured at 20 meters above ground level at the two airports. This increase in wind shear at 50 meters and 100 meters above ground level is as a result of decrease in frictional resistance which is more pronounced close to the ground. This explains that wind shear increases with altitude. 


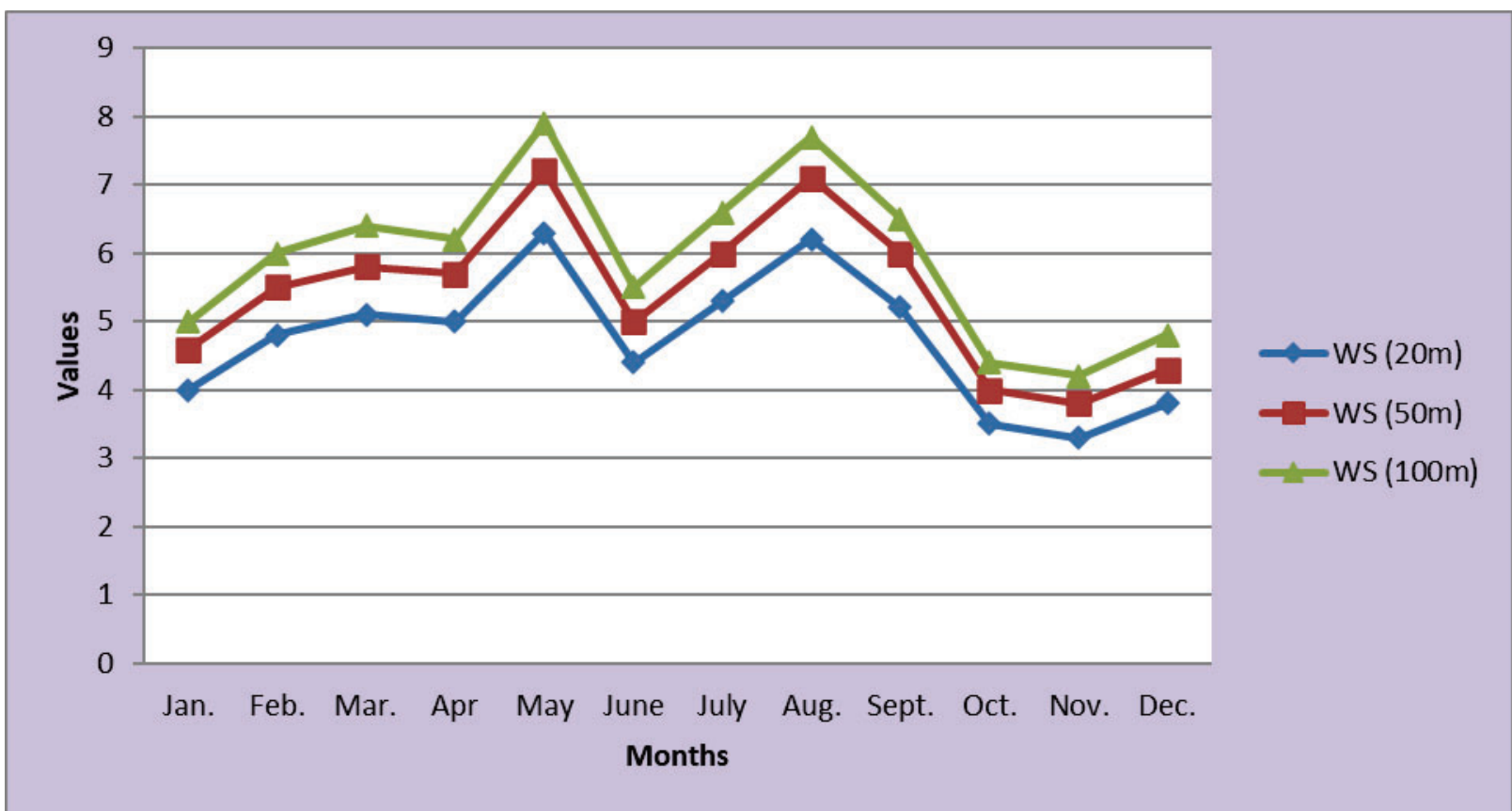

Fig. 3.1: Graphical representation of wind shear at $20 \mathrm{~m}, 50 \mathrm{~m}$ and $100 \mathrm{~m}$ above ground level at Murtala Mohammed International Airport, Lagos (2008-2018)

Source: Authors' computation

As shown in figure 3.1 , the distribution of wind shear at $20 \mathrm{~m}, 50 \mathrm{~m}$, and $100 \mathrm{~m}$ above ground level at Murtala Mohammed International Airport, Lagos from 2008-2018 follows similar pattern. In the first quarter of every year, the wind shear rises steadily and smoothly. Stepping into the first month of the second quarter, the wind shear spikes up and dropped sharply in the second month, rises again in July and August and decline steadily through to November, then with a rising attempt in December.

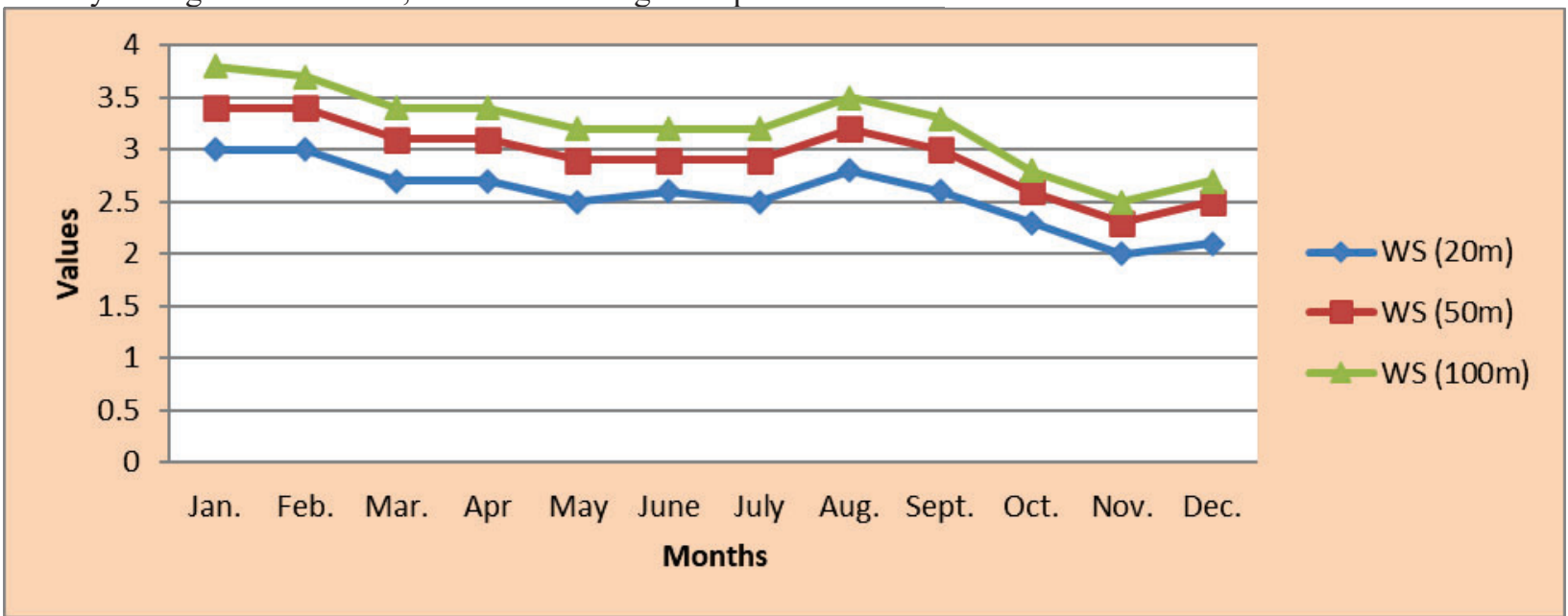

Fig. 3.2: Graphical representation of wind shear at $20 \mathrm{~m}, 50 \mathrm{~m}$ and $100 \mathrm{~m}$ above ground level at Port Harcourt International Airport (2008-2018)

Source: Authors' computation

The result in figure 3.2 shows that the distribution of wind shear at $20 \mathrm{~m}, 50 \mathrm{~m}$, and $100 \mathrm{~m}$ above ground level at Port Harcourt International Airport from 2008-2018 follows similar pattern. As shown in the result, the wind shear drops smoothly from January through July for the period. Approaching the month of August, it rose and after then decline continuously through to November; and rose slightly again in December. 


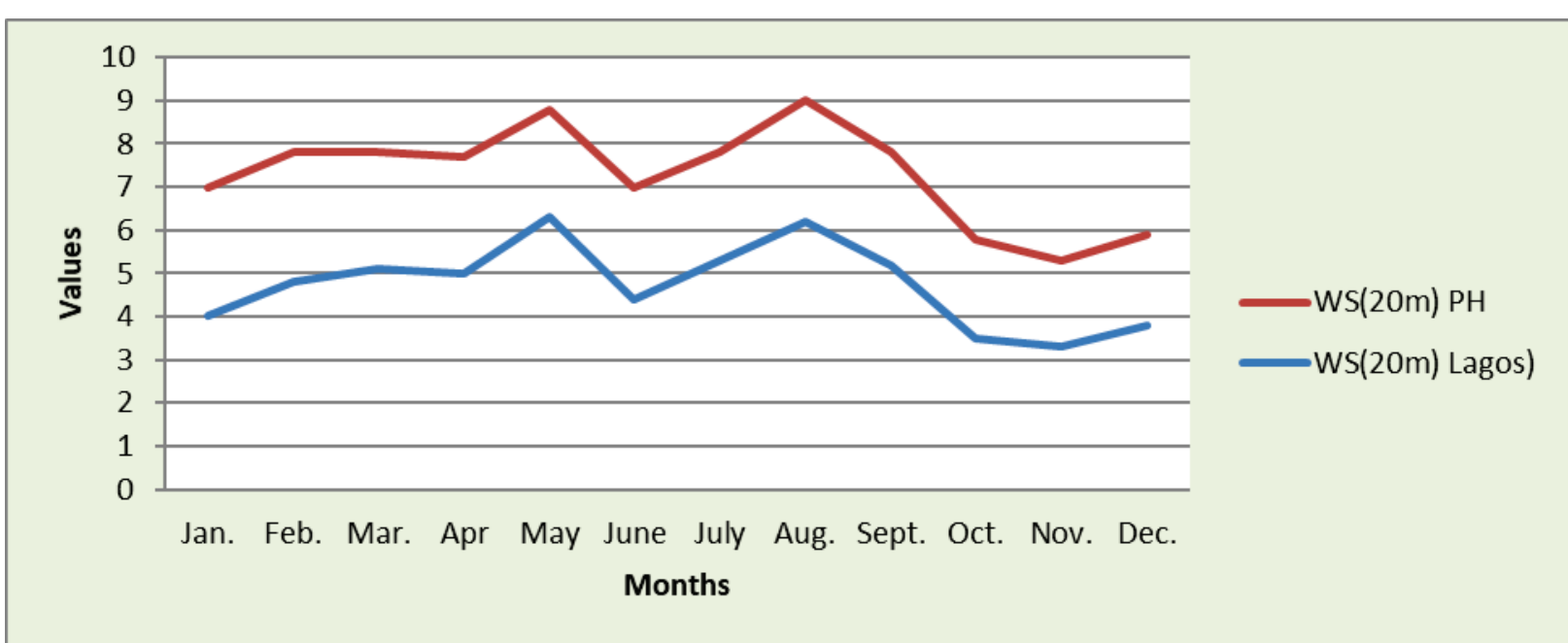

Fig. 3.3: Comparative graphical representation of wind shear measured at $20 \mathrm{~m}$ above ground level at Murtala Mohammed International Airport, Lagos and Port Harcourt International Airport (2008-2018) Source: Authors' computation

Comparatively, as presented in figure 3.3, the pattern of wind shear measured at $20 \mathrm{~m}$ above ground level at both Murtala Mohammed International Airport, Lagos, and Port Harcourt International Airport, Port Harcourt are very similar. Though the wind shear measured at $20 \mathrm{~m}$ are higher in Lagos compared to Port Harcourt international Airport, Nigeria.

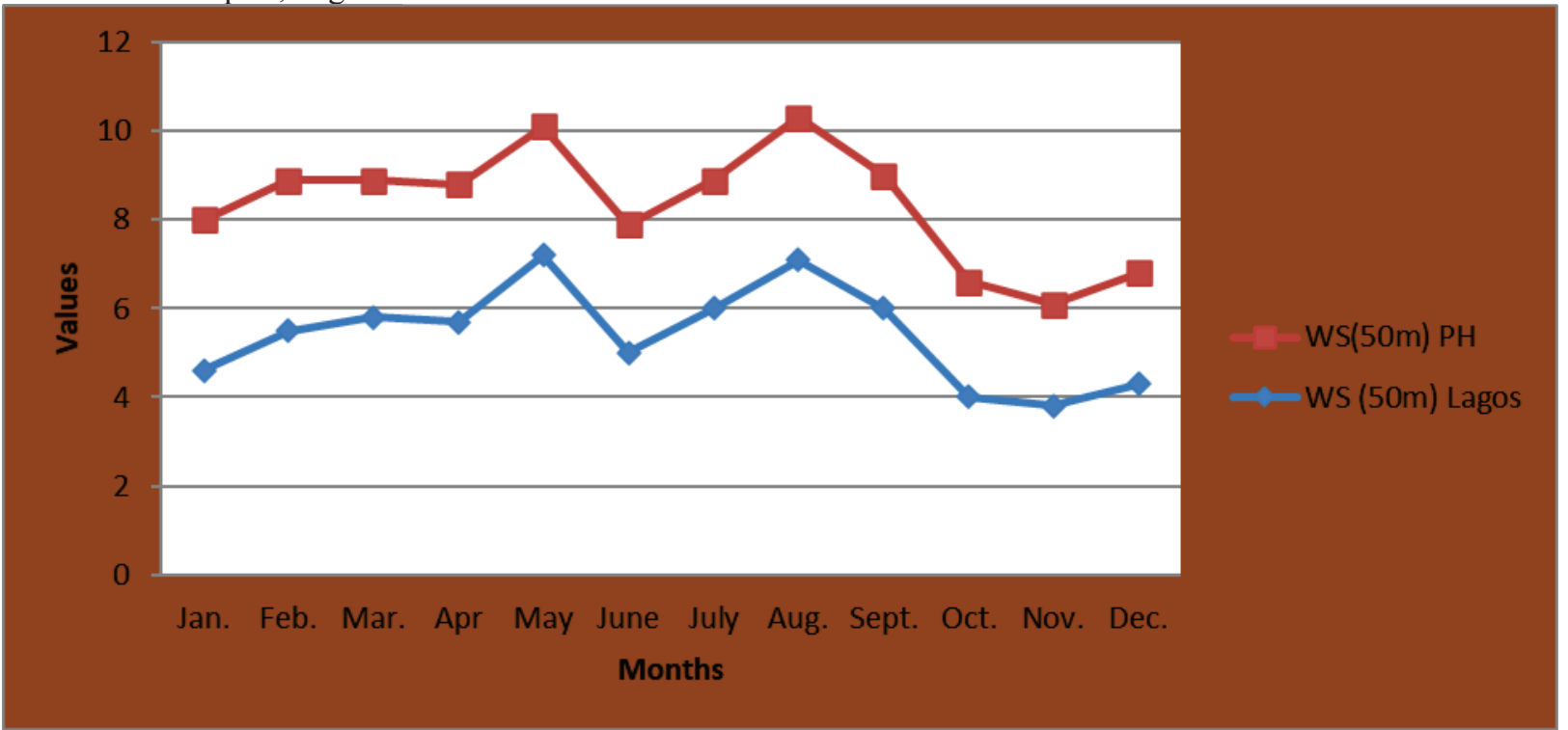

Fig. 3.4: Comparative graphical representation of wind shear extrapolated at 50m above ground level at Murtala Mohammed International Airport, Lagos and Port Harcourt International Airport (2008-2018) Source: Authors' computation

The graphical representation in figure 3.4 shows a similar pattern in distribution of wind shear extrapolated at 50m above ground level at Murtala Mohammed International Airport and Port Harcourt International Airport. From the graph, there is a smooth rise from January to March while the wind shear stood still through April. In May, it rose sparingly and dropped sharply in June. From June through August, the wind shear rose steadily and falls continuously from September to November. A little appreciation in the wind shear value was seen in December. 


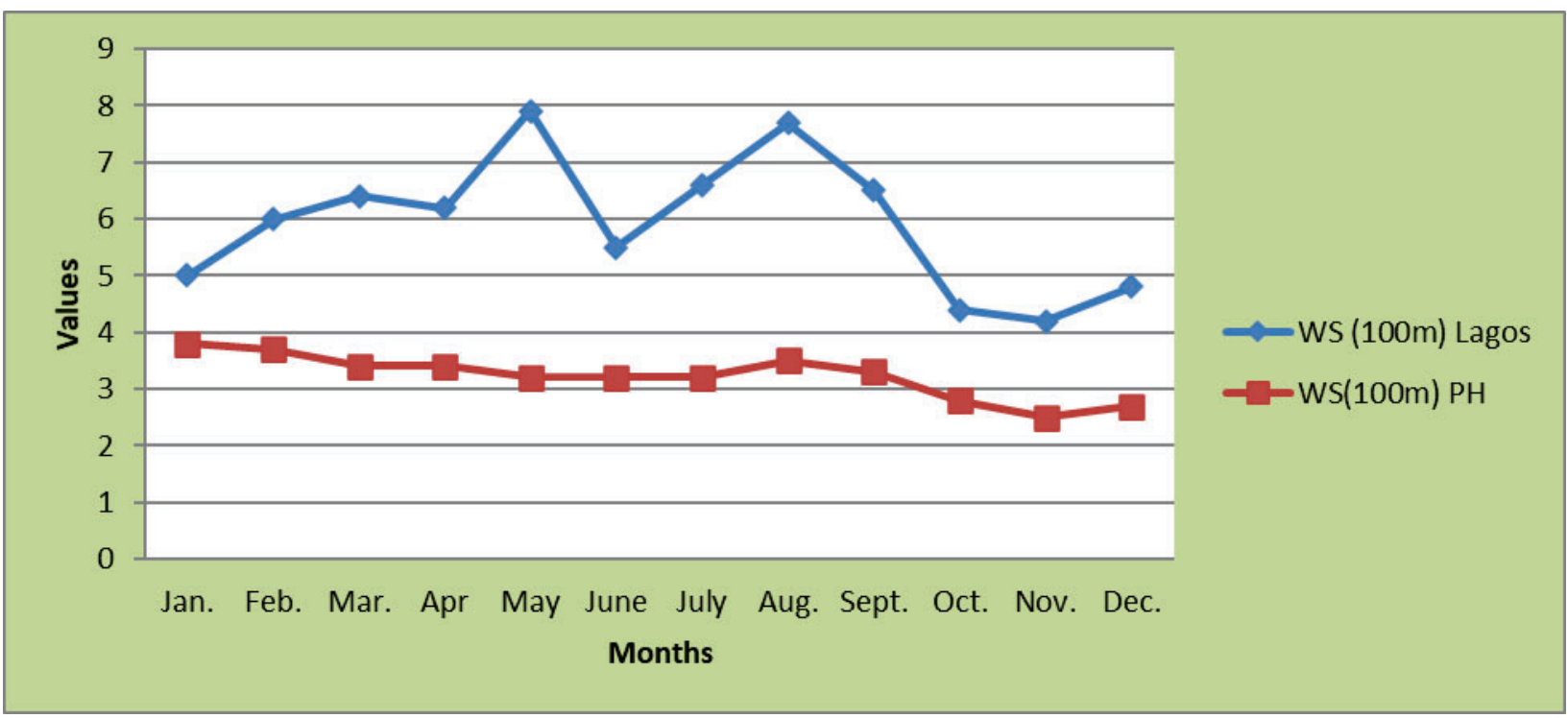

Fig. 3.5: Comparative graphical representation of wind shear extrapolated at $100 \mathrm{~m}$ above ground level at Murtala Mohammed International Airport, Lagos and Port Harcourt International Airport (2008-2018) Source: Authors' computation

As shown in figure 3.5 , there is dissimilarity in the patterns of wind shear extrapolated at $100 \mathrm{~m}$ above ground at Murtala Mohammed International Airport and Port Harcourt International Airport for the study period. Specifically, the wind shear distribution at Murtala Mohammed International Airport follows a random (zig-zag) pattern while the distribution at Port Harcourt International Airport is smooth, and detrended over the period. In a more specific term, the wind shear at Murtala Mohammed international Airport is steadily increasing between January and April, while from May through December, the wind shear continues to exhibit rise and fall movement.

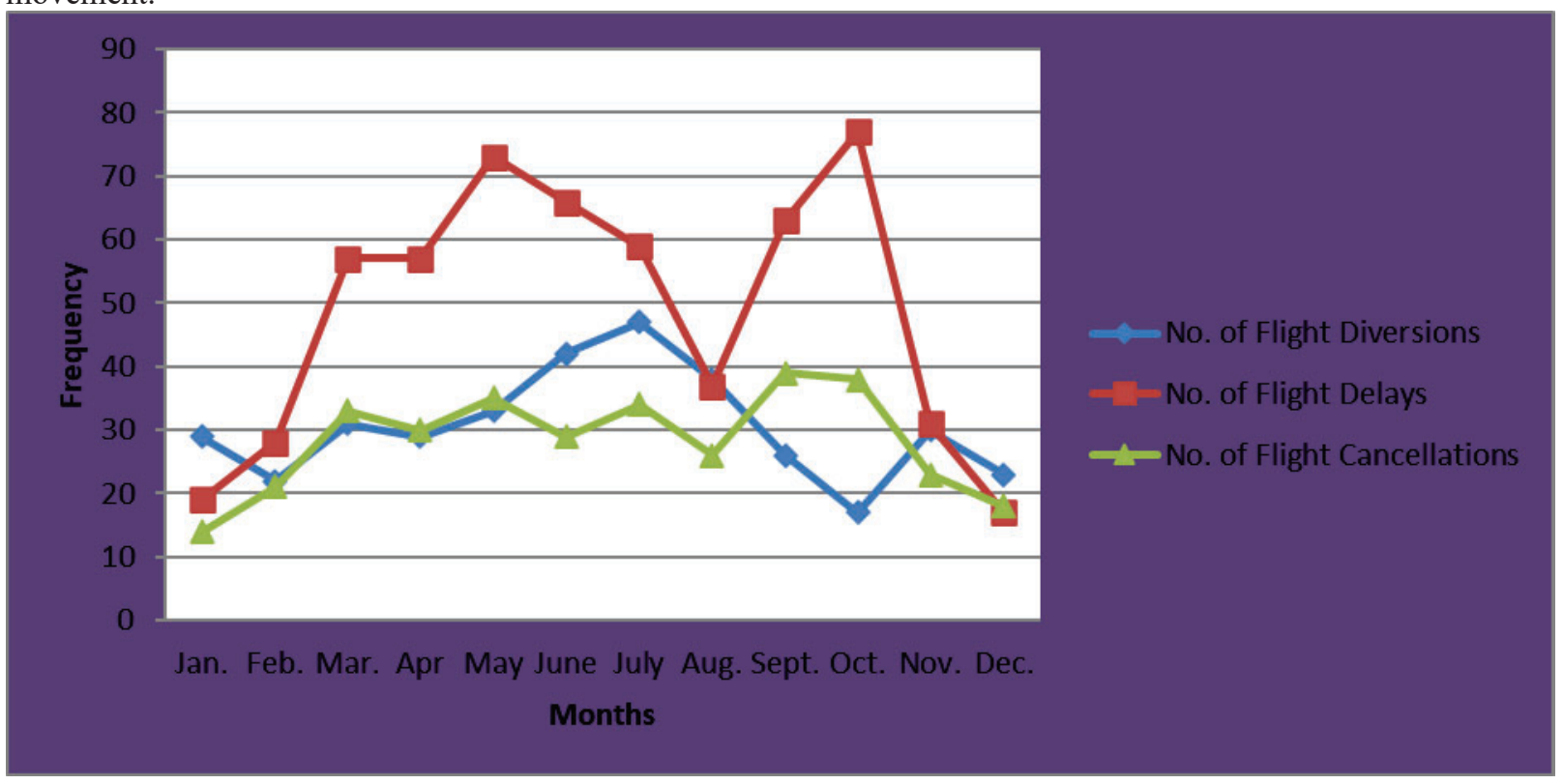

Fig. 3.6: Graphical representation of number of flight delays, cancellations and diversions at Murtala Mohammed International Airport, Lagos (2008-2018)

Source: Authors' computation

The pattern of occurrence of flight diversions, delays and cancellations at Murtala Mohammed International Airport, Lagos is random and unpredictable. Saliently, the distribution of number of flight cancellations over the period (2008-2018) shows similarity with the distribution of number of flight delays within the period. The implication is that, as the number of flight delays increases, the number of flight cancellations increases, and vice versa. This agreement as shown in figure 3.6 did not extend fully to frequency of flight diversions in the Airport; thereby indicating that flight delay or cancellation does not at all times lead to flight diversion. 


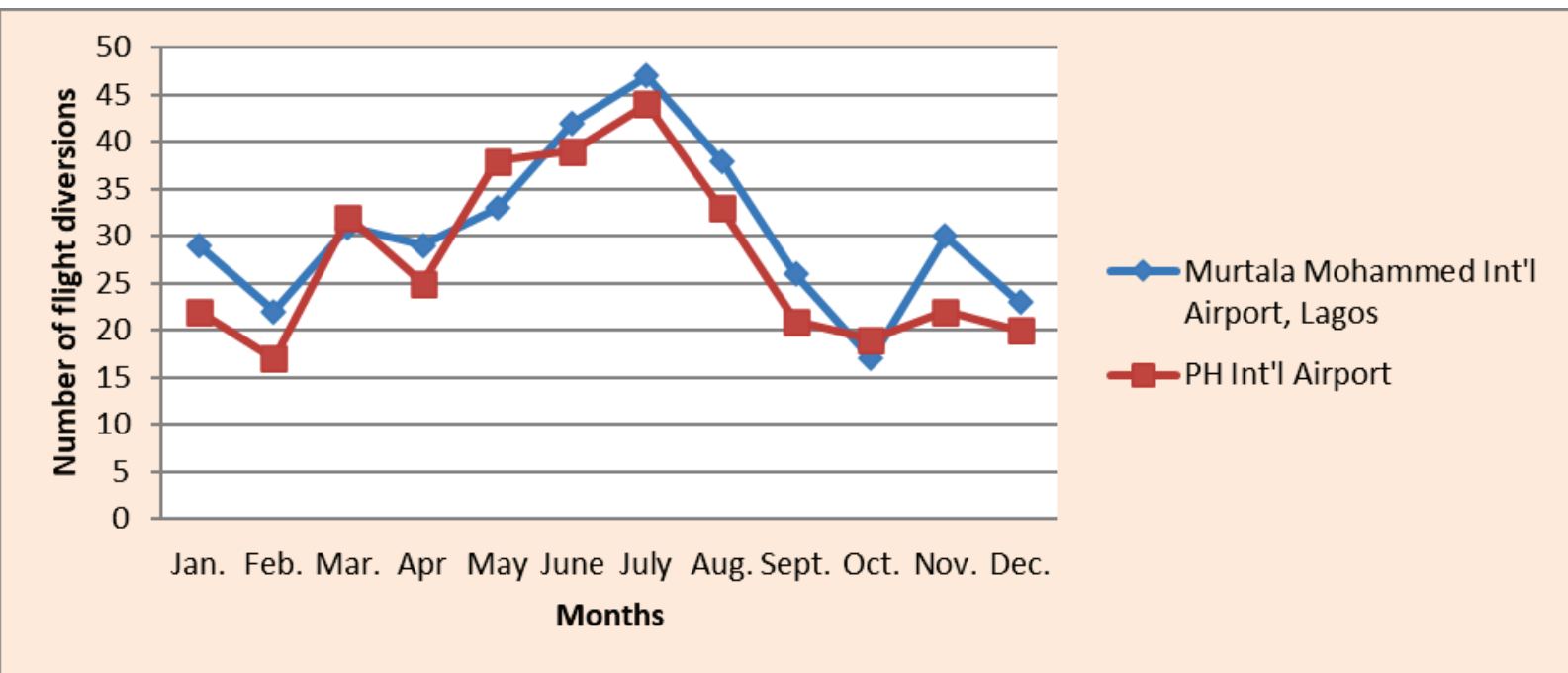

Fig. 3.7: Comparative graphical representation of number of flight diversions at Murtala Mohammed International Airport, Lagos and Port Harcourt International Airport (2008-2018)

\section{Source: Authors' computation}

As shown in figure 3.7, there is a confirmed similar distribution in frequency of flight diversions at Murtala Mohammed International Airport and Port Harcourt International Airport, though with a slightly varying magnitude which is traceable to difference in location. The both exhibit a random (zig-zag) movement for the period.

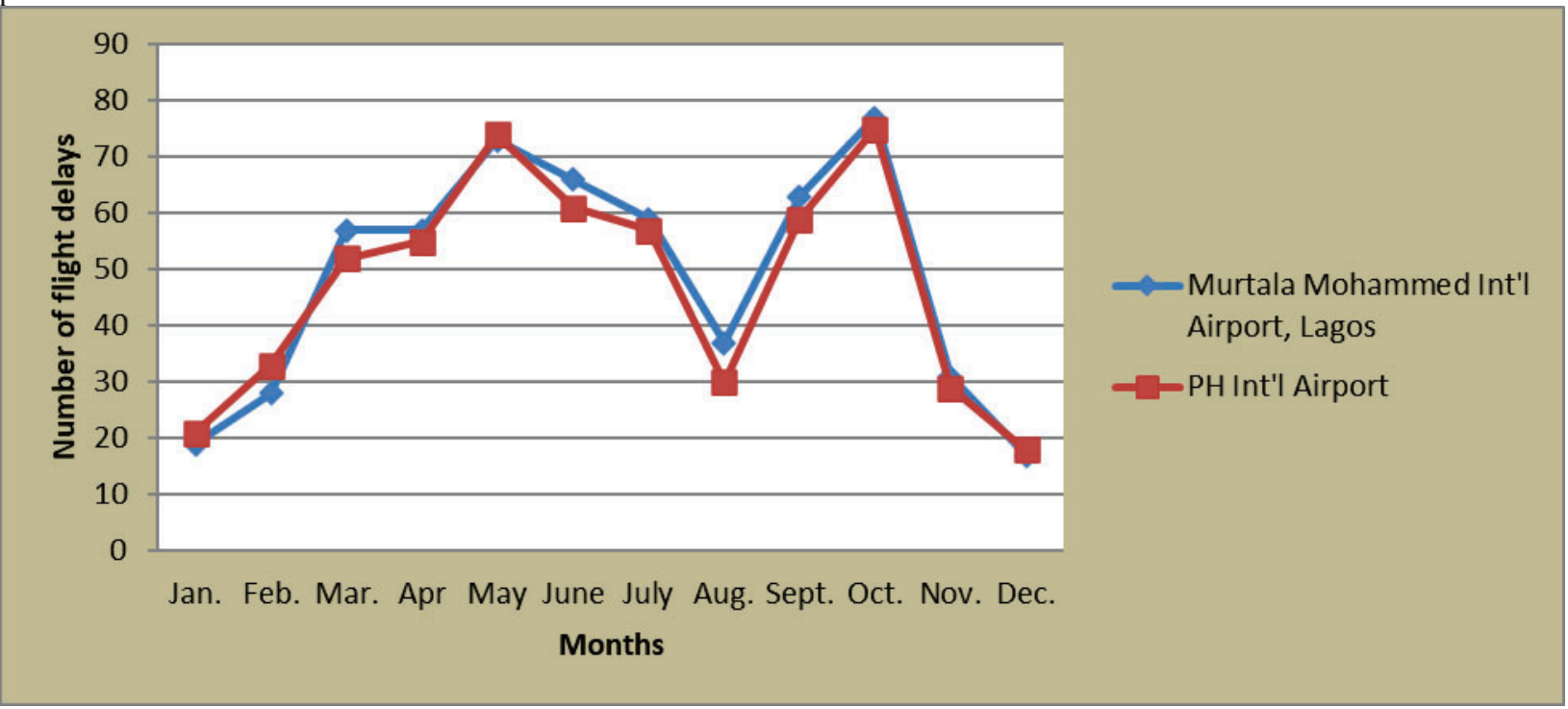

Fig. 3.8: Comparative graphical representation of number of flight delays at Murtala Mohammed International Airport, Lagos and Port Harcourt International Airport (2008-2018)

\section{Source: Authors' computation}

The graphical representation in figure 3.8 exposed the close relationship in the distribution of number of flight delays at Murtala Mohammed International Airport and Port Harcourt International Airport. Evidently, there is almost a perfect relationship in frequency of flight delays in both Airports at the two locations in Nigeria. 


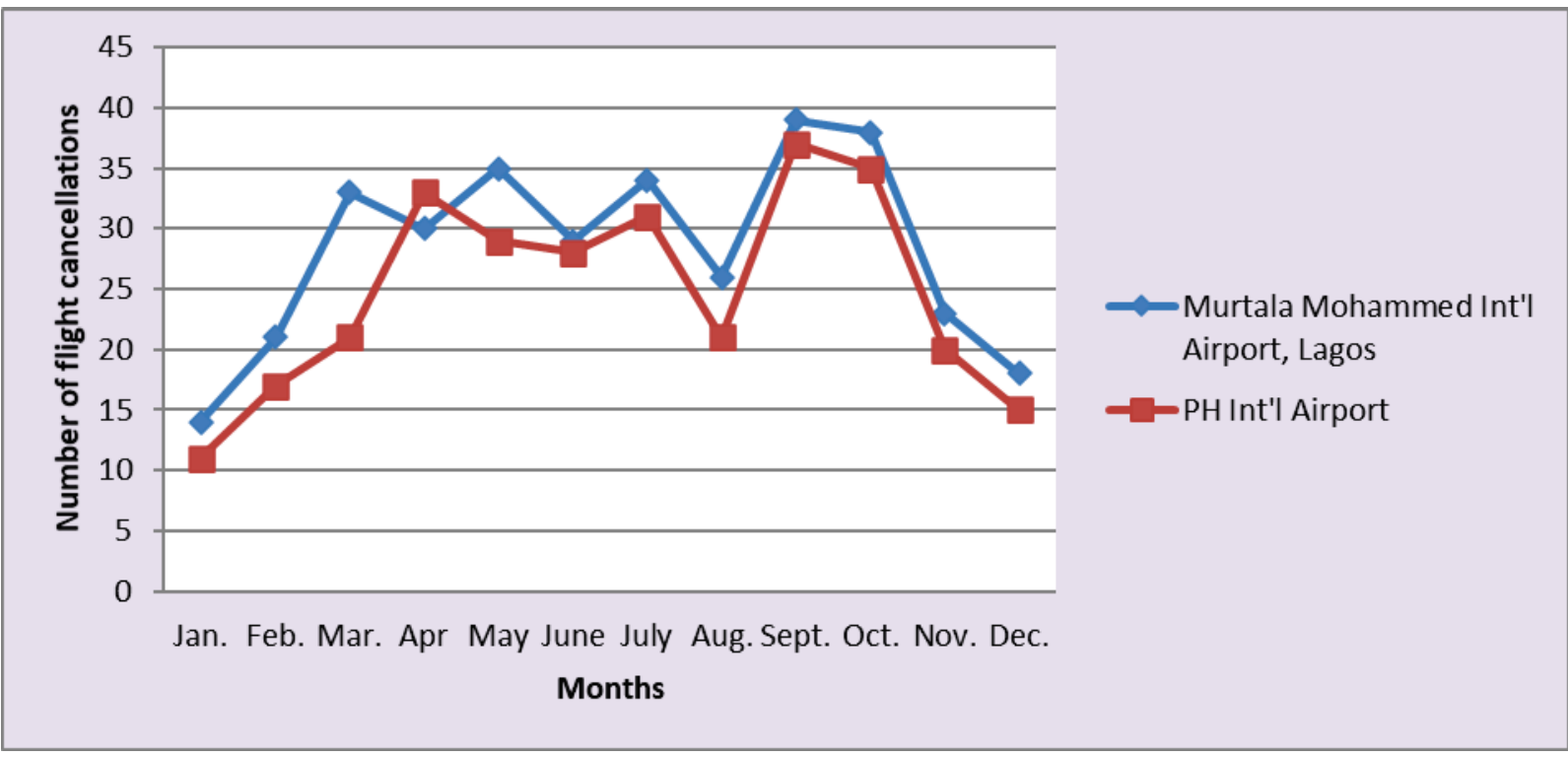

Fig. 3.9: Comparative graphical representation of number of flight cancellations at Murtala Mohammed International Airport, Lagos and Port Harcourt International Airport (2008-2018)

Source: Authors' computation

The comparative graphical representation of the number of flight cancellations at Murtala Mohammed International Airport and Port Harcourt International Airport shows that at the early months of every year (January through March), the number of flight cancellation increases steadily at both Airports. In April through May, the frequency of flight cancellations at the both Airports is in counter pattern (one rises and the other falls). From June through December, the pattern of flight cancellations at both Airports aligns with each other.

\subsection{Determining the Airport Safer For Landing}

Based on the wind shear movement and number of flight diversions, delays and cancellations associated with it, the Airport safer for landing was determined statistically as follows:

Table 3.9: Airport Safer for Landing

\begin{tabular}{l|llll|llll} 
& \multicolumn{3}{|l}{ Murtala Mohammed Int'l Airport } & \multicolumn{5}{|l}{ Port Harcourt Int'l Airport } \\
\cline { 2 - 9 } Parameters & WS & FDS & FDL & FCL & WS & FDS & FDL & FCL \\
\hline Mean & 5.36 & 30.58 & 48.67 & 28.33 & 2.91 & 27.67 & 47.00 & 24.83 \\
Std. dev. & 1.10 & 8.54 & 21.12 & 8.03 & 0.35 & 9.11 & 19.93 & 8.45 \\
CV & $20.5 \%$ & $27.9 \%$ & $43.4 \%$ & $28.3 \%$ & $12.0 \%$ & $32.9 \%$ & $42.4 \%$ & $34.0 \%$ \\
\hline
\end{tabular}

$\mathrm{CV}=$ Coefficient of variation

From the coefficient of variation (CV) results above, Port Harcourt International Airport (with overall higher CV) is considered safer for flight landing.

\section{Conclusion and Recommendations}

The study shows that wind shear increases with altitude at the study areas. This is evident as the values of wind shear extrapolated at $50 \mathrm{~m}$ and $100 \mathrm{~m}$ above ground level at the airports are higher than the values of wind shear measured at $20 \mathrm{~m}$ above ground level at the airports. This increase in wind shear at $50 \mathrm{~m}$ and $100 \mathrm{~m}$ above ground level is as a result of decrease in frictional resistance which is more pronounced closed to the ground.

A similarity in the distribution pattern of wind shear at $20 \mathrm{~m}, 50 \mathrm{~m}$ and $100 \mathrm{~m}$ above ground level at Murtala Mohammed International Airport was revealed by the study. Likewise, at Port Harcourt International Airport, wind shear at $20 \mathrm{~m}, 50 \mathrm{~m}$ and $100 \mathrm{~m}$ above ground level also shows a similarity in the distribution pattern. Comparatively, the distribution pattern of wind shear at $20 \mathrm{~m}$ and $50 \mathrm{~m}$ above ground level at the two airports are very similar, though the wind shear measured at $20 \mathrm{~m}$ are higher in Lagos compared to Port Harcourt International Airport, Nigeria. The distribution patterns of wind shear extrapolated at $100 \mathrm{~m}$ above ground level at the airports show some dissimilarities. The wind shear at Murtala Mohammed International Airport has a random (zig-zag) distribution whereas wind shear at Port Harcourt International Airport has smooth distribution and detrended over the period.

The study reveals that the number of flight delays increases as the number of flight cancellations increase, and vice versa. It also reveals that flight delays or cancellations do not always lead to flight diversions in the study areas. The study also reveals based on the effects of wind shear and its resultant flight delays, diversions and cancellations that Port Harcourt International Airport with overall higher coefficient of variation (CV) is 
considered safer for flight landing.

The study, from its findings, has shown the months with high rate of wind shear in the study areas, the knowledge of this can help the Meteorologists and Pilots in the forecast and piloting duties respectively. It therefore, recommends subsequent training and retraining of Meteorologists, Pilots and Air Traffic Controllers on wind shear and related weather issues. The work also recommends that more studies should be carried out by the students and researchers on wind shear. This is to help the aviation industry especially in the developing countries, like Nigeria, to grow to full maturity, and to curb accidents caused by wind shear.

\section{References}

Abdulla, A.O. (2014). Wind analysis and estimate of the wind shear exponent of the Sulaymanyiah International Airport Area. M.Sc. Thesis, University of Sulaimani, Sulaimani, Iraq.

Ajie, U. E., \& Dienye, A. (2014). Spatial data analysis of solid waste management system in Port Harcourt Metropolis after 100 years of its existence. FIG Congress, Kuala Lumpur, Malaysia.

Azad, K. A. (2011). Effect of wind shear on wind velocity at coastal sites of Bangladesh. Proceedings of the international conference on Mechanical Engineering. Dhaka, Bangladesh.

Building Nigeria's Response to Climate Change Project (BNRCC) (2012). Towards a Lagos State climate change adaptation strategy. Report prepared for the Honourable Commissioner of Environment, Lagos State.

Demographia (2016). Demographia World Urban Areas.

Enete, I. C., Ajator, U., \& Nwoko, K. C. (2015). Impacts of thunderstorm on flight operations in Port-Harcourt International Airport, Omagwa, Rivers State, Nigeria. International Journal of Weather, Climate Change and Conservation Research, 1(1), $1-10$.

Ezenwaji, E. E. \& Chima, G. N. (2016). Rest of Africa. In: Ezenwaji, E. E., Enete, I. C., \& Anyaeze, E. U. (Eds.). General Geography for Schools and Colleges. Awka: SCOA Heritage Nigeria Limited.

Iloeje, N. P. (1979). A new geography of West Africa. Longman Group Limited, Nigeria.

Jones, B. (2004a). Space weather effects on aircraft operations. Proceedings of the NATO advanced research workshop on effects of space weather on technology infrastructure, Rhodes, Greece, 25-29 March 2003, Kluwer Vol II/176, 215-234.

Knecht, W. R. (2008). Use of weather information by general aviation pilots, Part II, Qualitative: Exploring factors involved in weather-related decision making. (Technical Report DOT/FAA/AM- 08/7).Washington, DC: Federal Aviation Administration, Office of Aerospace Medicine.

Mathew, S. (2006). Wind energy fundamentals, resource analysis and economics. Kerala: Springer.

Merki, J. P. (1970). Structural geology of the cenezoic Niger Delta. African Geology, University of Ibadan Press. Pp. $251-268$.

Murat, R. C. (1970). Stratigraphy and paleography of the cretaceous and lower teriary in Southern Nigeria. In: Dessauvagie, T. T. J. and Whiteman, A. J. (Eds.). African Geology, University of Ibadan, Nigeria. Pp. 251 $-266$.

Musa, M. C. (2014). Effects of some weather parameters on flight operations in Kano and Abuja International Airports, Nigeria. Unpublished M.Sc. Thesis, Department of Geography, Ahmadu Bello University, Zaria.

National Bureau of Statistics of Nigeria (2013).

National Oceanic and Atmospheric Administration (2018). Port Harcourt climate normals 1961 - 1990.

National Research Council (1983). Low altitude wind shear and its hazard to aviation. Washington DC: National Academy Press.

Nwankwoala, H. O., \& Walter, I. O. (2012). Assessment of groundwater quality in shallow coastal aquifers of Okrika Island, Eastern Niger Delta, Nigeria. Ife Journal of Science, 14(2), 297 - 304.

Nwankwoala, H. O., Abam, T. K. S., Ede, P. N., Teme, S. C., \& Udom, G. J. (2008). Estimates of aquifer hydraulic properties using pumping test data: A case study of Port Harcourt and Environs. Water Resources Journal of the Nigerian Association of Hydrogeologists, 18, 25 - 31.

Onwuadiochi, I. C., Ijioma, M. A., \& Ozoemene, M. L. (2020). Comparative analysis of the effects of measured and calculated wind shear on aircraft operations in Sam Mbakwe Airport, Imo State, Nigeria. Environmental Review, 7(1).

Onwuadiochi, I. C., Ijioma, M. A., Ezenwaji. E. E., \& Obikwelu, M. C. (2020). Effects of wind shear on flight operations in Sam Mbakwe Airport, Imo State, Nigeria. Tropical Built Environment Journal, 7(1).

Oteri, A. U. (2013). Coastal groundwater resources, abstraction, quality and related environmental concerns. www.lagoswaterreg.org.ng/events.php? $\mathrm{p}=191$.

Oteri, A. U., \& Ayeni, R. A. (2016). The Lagos megacity. Water, megacities and global change.

Reyment, R. A. (1965). Aspects of the geology of Nigeria. Ibadan: University of Ibadan Press.

Roberto, B. (2014). Analysis of wind data for airport runway design. Journal of Airline and Airport Management, $4(2), 97-116$.

Sasse, M. \& Hauf, T. (2003). A study of thunderstorm-induced delays at Frankfurt Airport, Germany. 
Meteorological Applications, 10(1), 21 - 30.

Short, K. C. \& Stauble, A. J. (1967). Outline geology of the Niger Delta. Journal of Mining Geology, 26(2), 279 -285 .

The Tide News (2013). PH, new hub for medical tourism. Port Harcourt: Rivers State Newspaper Corporation.

United Nations Cetefor Human Settlements (UNCHS-Habitat) (1996). An urbanizing World - Global report on human settlements, pp 11-24.

Wagner, W., Courtney, M., Gottschall, J., \& Marsden, P. L. (2011). Accounting for the speed shear in wind turbine power performance measurement. Roskilde, Denmark: Wiley Online Library.

Weli, V. E. \& Emenike, G. C. (2016). Turbulent weather events and aircraft operations: Implications for aviation safety at the Port Harcourt international airport, Nigeria. International Journal of Weather, Climate Change and Conservation Research, 2(3), $19-30$.

Weli, V. E. \& Ifediba, U. E. (2014). Poor weather and flight operations: Implications for air transport hazard management in Nigeria. Ethiopian Journal of Environmental Studies and Management, 7(3), 235 - 243.

World Meteorological Organization (2018). World Weather Information Service - Port Harcourt. 